\title{
Estimating Household Fuel Oil/Kerosine, Natural Gas, and LPG Prices by Census Region
}

by D.A. Poyer and A.P.S. Teotia

Energy Systems Division,

Argonne National Laboratory, 9700 South Cass Avenue, Argonne, Illinois 60439

August 1994

Work sponsored by United States Department of Energy,

Office of Minority Economic Impact

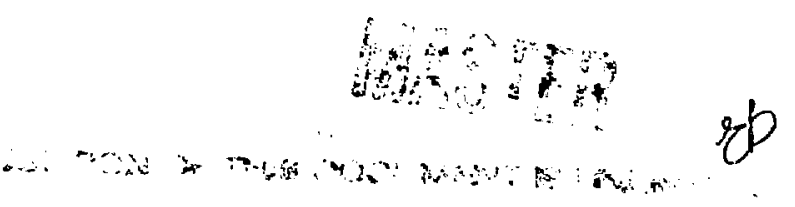




\section{Contents}

Acknowledgments.................................................................... vi

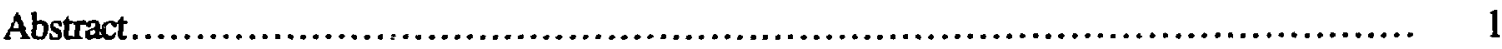

1 introduction............................................................................ 1

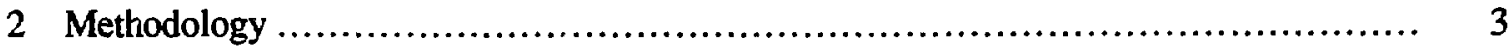

3 Estimation of Fuel-Share and Fuel-Price Models ............................... 8

3.1 Fuel Oil/Kerosine Share and Price Models.................................. 8

3.1.1 Northeast Census Region.............................................. 8

3.1.2 Midwest Census Region ........................................... 8

3.1.3 South Census Region .............................................. 8

3.1.4 West Census Region .................................................. 14

3.2 Natural Gas Share and Price Modeis............................................. 14

3.2.i Northeast Census Region.............................................. 14

3.2.2 Midwest Census Region................................................ 14

3.2.3 South Census Region ............................................. 21

3.2.4 West Census Region ................................................ 21

3.3 Liquefied Petroleum Gas Share and Price Models.............................. 21

3.3.1 Northeast Census Region.............................................. 21

3.3.2 Midwest Census Region...................................................... 28

3.3.3 South Census Region ................................................. 28

3.3.4 West Census Region ........................................... 28

4 Overview of Fuel-Share Models.................................................. 36

5 Overview of Fuel-Price Models.................................................... 38

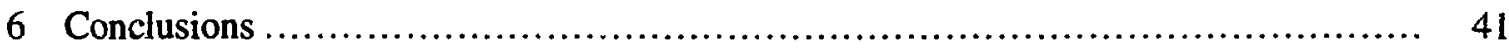

6.1 Fuel-Share Models .......................................................... 41

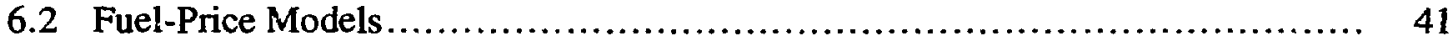

7 References ......................................................................... 43

\section{Tables}

1 Maximum-Likelihood-Parameter Estimates for Fuel Oil/Kerosine Fuel-

Share Model: Northeast Census Region.

2 Ordinary-Least-Squares-Parameter Estimates for Fuel Oil/Kerosine

Price Model: Northeast Census Region

3 Maximum-Likelihood-Parameter Estimates for Fuel Oil/Kerosine

Fuel-Share Model: Midwest Census Region. 


\section{Tables (Cont.)}

4 Ordinary-Least-Squares-Parameter Estimates for Fuel Oil/Kerosine

Price Model: Midwest Census Region.............................................

5 Maximum-Likelihood-Parameter Estimates for Fuel Oil/Kerosine

Fuel-Share Model: South Census Region.

6 Ordinary-Least-Squares-Parameter Estimates for Fuel Oil/Kerosine

Price Model: South Census Region.

7 Maximum-Likelihood-Parameter Estimates for Fuel Oil/Kerosine

Fuel-Share Model: West Census Region

8 Ordinary-Least-Squares-Parameter Estimates for Fuel Oil/Kerosine

Price Model: West Census Region ...

9 Maximum-Likelihood-Parameter Estimates for Natural Gas Fuel-

Share Model: Northeast Census Region....

10 Ordinary-Least-Squares-Parameter Estimates for Natural Gas Price Model:

Northeast Census Region

11 Maximum-Likelihood-Parameter Estimates for Natural Gas Fuel-Share

Model: Midwest Census Region

12 Ordinary-Least-Squares-Parameter Estimates for Natural Gas Price Model:

Midwest Census Region

13 Maximum-Likelihood-Parameter Estimates for Natural Gas Fuel-Share

Mode1: South Census Region

14 Ordinary-Least-Squares-Parameter Estimates for Natural Gas Price Model:

South Census Region.

15 Maximum-Likelihood-Parameter Estimates for Natural Gas Fuel-Share

Model: West Census Region

16 Ordinary-Least-Squares-Parameter Estimates for Natural Gas Price Model:

West Census Region.

17 Maximum-Likelihood-Parameter Estimates for LPG Fuel-Share Model:

Northeast Census Region.

18 Ordinary-Least-Squares-Parameter Estimates for LPG Price Model:

Northeast Census Region.

19 Maximum-Likelihood-Parameter Estimates for LPG Fuel-Share Model:

Midwest Census Region

20 Ordinary-Least-Squares-Pararneter Estimates for LPG Price Model:

Midwest Census Region 


\section{Tables (Cont.)}

21 Maximum-Likelihood-Parameter Estimates for LPG Fuel-Share Model:

South Census Region............................................................... 32

22 Ordinary-Least-Squares-Parameter Estimates for LPG Price Model:

South Census Region.............................................................. 33

23 Maximum-Likelihood-Parameter Estimates for LPG Fuel-Share Model:

West Census Region.

24 Ordinary-Least-Squares-Parameter Estimates for LPG Price Model:

West Census Region............................................................ 35

25 Key Variables Significant at 5\% Level in Fuel-Share Models...................... 37

26 Key Variables Significant at 5\% Level in Fuel-Price Models ................... 39 


\section{Acknowledgments}

The athors of this report are pleased to acknowledge the assistance and advice of a number of people. This work was sponsored by the U.S. Department of Energy (DOE), and the support of the DOE research program manager, Georgia Johnson, is gratefully acknowledged. External review and comments were provided by Kenneth Train, Chairman, Department of Economics, University of California at Berkeley. We also wish to thank Vicki Skonicki for typing this report and Robert Putnam for editing it. 


\title{
Estimating Household Fuel Oil/Kerosine, Natural Gas, and LPG Prices by Census Region
}

by

\author{
D.A. Poyer and A.P.S. Teotia
}

\begin{abstract}
The purpose of this research is to estimate individual fuel prices within the residential sector. The data from four U.S. Department of Energy, Energy Information Administration, residential energy consumption surveys were used to estimate the models. For a number of important fuel types - fuel oil, natural gas, and liquefied petroleum gas - the estimation presents a problem because these fuels are not used by all households. Estimates obtained by using only data in which obseryed fuel prices are present would be biased. A correction for this selfselection bias is needed for estimating prices of these fuels. A literature search identified no past studies on application of the selectivity model for estimating prices of residential fuel oil/kerosine, natural gas, and liquefied petroleum gas. This report describes selectivity models that utilize the Dubin/McFadden correction method for estimating prices of residential fuel oil/kerosine, natural gas, and liquefied petroleum gas in the Northeast, Midwest, South, and West census regions. Statistically significant explanatory variables are identified and discussed in each of the models. This new application of the selectivity model should be of interest to energy policy makers, researchers, and academicians.
\end{abstract}

\section{Introduction}

The purpose of this research is to estimate individual energy prices used in estimation of an energy-demand system and to estimate fuel shares within end-use service categories. For a number of important fuel types - fuel oil, natural gas, and liquefied petroleum gas (LPG) - the estimation presents an intriguing problem because these fuels are not used by all households. As a result, the researcher is confronted with a censored sample due to self-selection. Estimates obtained by using only data based on observed fuel price would be biased. For this reason, a correction of the self-selection bias is needed for estimating fuel prices. A literature search was conducted to identify any past studies that might have corrected for this self-selection bias in estimating residential energy prices. No such study was identified. This report discusses the selectivity models developed for estimating individual prices paid for fuel oil/kerosire, natural gas, and LPG in the Northeast, Midwest, South, and West census regions. These models, based on a logit form of the choice equation, utilize the Conditional Expectation Correction Method described by Dubin and McFadden in an article in Econometrica.: 
These models ultimately will be used as instrumental variables in the space and water heating share models being developed at Argenne National Laboratory (ANL). The data from four U.S. Department of Energy, Energy Information Administration (DOE/EIA), residential energy consumption surveys (RECS) ${ }^{2-5}$ were used io estimate the models. Several reports on each of these surveys are available from DOE/EIA, including two key reports: Housing Characteristics, $1987^{6}$ and Household Energy Consumption and Expenditures, 1987.7

Note that the unit of analysis in this report is the household. 


\section{Methodology}

Subsequent to the pioneering approach of Heckman, 8 the self-selectivity problem has been analyzed by a number of academicians and researchers. Several models with self-selectivity have been discussed in detail by Maddala. ${ }^{9}$ Selectivity models have been applied in many types of studies: participation in the labor force, retirement decisions, returns to education, effects of unions on wages, effects of employment services, migration and income, physician behavior, tenure choice and demand for housing, and electric utility rates. (References on these studies can be found in Reference 9, page 289.) Engile noted that the inclusion of mills ratio is a distinctive feature of much of the work of self-seiectivity. 10

Our literarure search did not identify any past studies on the application of any selectivity model for estimating household prices for fuel oil/kerosine, natural gas, and L.PG, the subject matter of underlying research.

The procedure for estimating models with correction for selection bias is adapted from the Conditional Expectation Correction Method described by Dubin and McFadden. ${ }^{1}$ Additional background information is also available in the related article, "Selection Bias in Linear Regression, Logit, and Probit Models," by Dubin and Rivers. "1

In the first step, a binary logit model for fuel share is estimated. The logit model gives the probability $(p)$ that the household uses a particular fuel. For the logit model, if a household uses a particular fuel (natural gas, fuel oil/kerosine, or LFG), the dependent variable (p) takes the value of 1 ; otherwise, it is 0 .

Within a census region, the selection of the fuel is affected by many household factors, such as personal preferences about end-use equipment/systerns (e.g., gas dryer vs. electric dryer), type of home (e.g., higher owner occupancy rates in single-family homes than in multifamily homes), age of home (e.g., availability of heating and cooling ducts, degree of thermal integrity of home), size of home (e.g., variation in square feet heated/cooled), and race/ethnicity of household (e.g., differences in housing patterns). Household variables are entered into the following logit model:

$$
\begin{gathered}
p=\frac{e^{w}}{1+e^{w}} \\
w=C_{0}+\sum_{i=1}^{i=k} C_{i} X_{i}
\end{gathered}
$$

where:

$$
\begin{aligned}
p & =\text { Probability of a particular fuel being used by a household } \\
C_{0} & =\text { Constant }
\end{aligned}
$$




$$
\begin{aligned}
C_{i} & =\text { Coefficient associated with the } i^{\text {th }} \text { variable } \\
X_{1} & =1981 \text { RECS durnmy variable } \\
X_{2} & =1982 \text { RECS dummy variable } \\
X_{3} & =1984 \text { RECS dummy variable } \\
X_{4} & =\text { Cooling degree days (CDD) } \\
X_{5} & =\text { Heating degree days (HDD) } \\
X_{6} & \left.=\text { Joint degree days ( } X_{4} \times X_{5}\right) \\
X_{7} & =\text { Real income } \\
X_{8} & =\text { Square feet heated } \\
X_{18} & =\text { Central city location dimmy variable } \\
X_{9} & =\text { Attached, single-family home dummy variable } \\
X_{17} & =\text { Year home built, } \geq 1980 \text { dummy variable } \\
X_{10} & =2-4 \text { multifamily units dummy variable } \\
X_{13} & =\text { Year home built, }<1940 \text { dummy variable } \\
X_{11} & =>4 \text { multifamily units dummy variable } \\
X_{12} & =\text { Mobile homes dummy variable } \\
X_{15} & =\text { Year home built, } 1960-74 \text { dummy variable } \\
X_{16} & \text { Year home built, } 1975-79 \text { dummy variable } \\
&
\end{aligned}
$$




$$
\begin{aligned}
X_{19} & =\text { Poor householder dummy variable } \\
X_{20} & =\text { Hispanic origin dummy variable } \\
X_{21} & =\text { Black householder dummy variable } \\
k & =\text { Number of explanatory variables }
\end{aligned}
$$

Because of singularity problems, not all of these variables were used in some cases. The maximum likelihood method was used for estimating fuel-share models. ${ }^{12}$

In the second step, these share (probability) models were then used to construct a mills ratio to serve as a selectivity correction term in the fuel price models. The mills ratio $(m)$ is:

$$
m=((1-p) \log (1-p) / p)+\log (p)
$$

In the third step, the average fuel price (PF) is estimated as a linear function of the selected variables and the mills ratio. The average fuel price for a household is calculated by dividing the annual bill by the quantity consumed. The parameters of fuel-price models were estimated by using ordinary least-squares (OLS) techniques. ${ }^{12}$ Within a census region, the average price a household pays for a fuel is affected by many household factors, such as location of household (e.g., state-by-state variation in natural gas rates approved by appropriate state commission, price variation in case of more than one gas utility in a state), type of home (e.g., different rate structures for single-family and multifamily homes in several states), age of home (e.g.. greater energyconsumption intensity in older, energy-inefficient homes), and race/ethnicity of houselold (e.g., cost of serving certain high-density ethnic population areas may be different for suppliers of LPG and fuel oil/kerosine). Hcusehold variables are entered into the following iinear model:

$$
P F=d_{0}+\sum_{j=1}^{j=n} d_{j} X_{j}+d_{n+1} m
$$

where:

$$
\begin{aligned}
& P F=\text { Average fuel price paid by a household } \\
& d_{0}=\text { Constant } \\
& d_{j}=\text { Coefficient associated with the } j^{\text {th }} \text { variable } \\
& x_{1}=1981 \text { RECS dummy variable }
\end{aligned}
$$




$$
\begin{aligned}
X_{2} & =1982 \text { RECS dummy variable } \\
X_{3} & =1984 \text { RECS dummy variable } \\
X_{4} & =\text { Cooling degree days } \\
X_{5} & =\text { Heating degree days } \\
X_{6} & =\text { Joint degree days }\left(X_{4} \times X_{5}\right) \\
X_{7} & =\text { Real income } \\
X_{8} & =\text { Square feet heated } \\
X_{9} & =\text { Attached, single-family home dummy variable } \\
X_{10} & =2-4 \text { multifamily units dummy variable } \\
X_{11} & =>4 \text { multifamily units duminy variable } \\
X_{12} & =\text { Mobile homes dummy variable } \\
X_{13} & =\text { Year home built, }<1940 \text { dummy variable } \\
X_{19} & =\text { Poor householder dummy variable } \\
X_{14} & =\text { Year home built, } 1940-49 \text { dummy variable } \\
X_{15} & =\text { Year home built, } 1960-74 \text { dummy variable } \\
X_{16} & =\text { Year home built, } 1975-79 \text { dummy variable } \\
X_{17} & =\text { Year home built, } \geq 1980 \text { dummy variable } \\
X_{18} & =\text { Central city location dummy variable } \\
X_{20} &
\end{aligned}
$$




$$
\begin{aligned}
X_{21} & =\text { Black householder dummy variable } \\
X_{22} & =\text { Consumer Price Index }- \text { Urban (CPIU) } \\
m & =\text { Mills ratio }
\end{aligned}
$$$$
d_{n+1}=\text { Coefficient associated with mills ratio }
$$$$
n=\text { Number of explanatory variables other than mills ratio }
$$

Once again, because of singularity problems, not all of these variables were used. 


\section{Estimation of Fuel-Share and Fuel-Price Models}

\subsection{Fuel Oil/Kerosine Share and Price Models}

\subsubsection{Northeast Census Region}

Table 1 shows the maximum-likelihood-parameter estimates for the fuel oil/kerosine fuelshare model in the Northeast census region. The fuel oil/kerosine model is specified as a logit funcition of 21 variables. Seven variables are significant at the 5\% level: homes built after 1979, attached single-family homes, mobile homes, homes built from 1960 through 1974, two-to-four multifamily homes, real income, and Hispanic ethnicity.

Table 2 shows the OLS-parameter estimates for the Northeast census region fuel oil/kerosine fuel-price model. The price model is specified as a linear function of 20 variables, including the mills ratio. Seven variables are significant at the 5\% level: the CPIU, larger-thanfour multifamily units, the 1984 RECS dummy variable, mobile homes, homes built from 1960 through 1974, attached single-family homes, and central city location.

\subsubsection{Midwest Census Region}

Table 3 shows the Midwest census region maximum-likelihood-parameter estimates for the fuel oil/kerosine fuel-share model. The parameter estimates associated with the 1982 RECS dummy variable, the 1981 RECS dummy variable, and larger-than-four multifamily units are significant at the $5 \%$ level.

Table 4 shows the OLS-parameter estimates for the Midwest census region fuel oil/kerosine price model. Nine variables are significant at the 5\% level: the CPIU, the 1984 RECS dumr.y variable, cooling degree days, the 1982 RECS dummy variable, mills ratio, attached single-family homes, Hispanic ethnicity, central city location, and homes built from 1960 through 1974.

\subsubsection{South Census Region}

Table 5 shows the South census region maximum-likelinood parameter estimates for the fuel oil/kerosine fuel-share model. In the model for the South census region, six variables are significant at the 5\% level: the 1981 RECS dummy variable, mobile homes, joint degree days (cooling degree days multiplied by heating degree days), the 1982 RECS dummy variable, homes built after 1979, and heating degree days. 
TABLE 1 Maximum-Likelihond-Parameter Estimates for Fuel Oil/Kerosine Fuel-Share Model: Northeast Census Region

\begin{tabular}{|c|c|c|c|c|c|}
\hline Variable & $\begin{array}{l}\text { Variable } \\
\text { Code }\end{array}$ & $\begin{array}{c}\text { Parameter } \\
\text { Estimate }\end{array}$ & $\begin{array}{c}\text { Standard } \\
\text { Error }\end{array}$ & Chi Square & Probability \\
\hline & Intercept & 0.1430 & 0.7617 & 0.04 & 0.85 \\
\hline 1981 RECS & D1981 & 0.0823 & 0.1711 & 0.23 & 0.63 \\
\hline 1982 RECS & D1982 & 0.1974 & 0.2109 & 0.88 & 0.35 \\
\hline 1984 RECS & D1984 & 0.1378 & 0.1713 & 0.65 & 0.42 \\
\hline Cooling degree days & CDD65 & 0.00092 & 0.000966 & 0.90 & 0.34 \\
\hline Heating degree days & HDL65 & $4.14 E-7$ & 0.000094 & 0.00 & 0.99 \\
\hline Joint degree days & CDD65*Hdd65 & $-7.321 E-8$ & $1.745 \mathrm{E}-7$ & 0.18 & 0.67 \\
\hline Real income & INCOME & -0.000011 & $4.421 E-6$ & 6.18 & 0.01 \\
\hline Square feet heated & HEATED & 0.0001 & 0.000078 & 1.79 & 0.18 \\
\hline Attached single-family home & SINGFAM2 & -0.7986 & 0.2451 & 10.62 & 0.00 \\
\hline 2-4 Multifamily units & MULTFAM1 & -0.5118 & 0.1896 & 7.29 & 0.01 \\
\hline$>4$ Multifamily units & MULTFAM2 & -0.1762 & 0.2086 & 0.71 & 0.40 \\
\hline Mobile homes & MOBHLOM & 1.1778 & 0.4054 & 8.44 & 0.04 \\
\hline Year home built, <1940 & KHOUS1 & -0.0747 & 0.1923 & 0.15 & 0.70 \\
\hline Year home built, $1940-49$ & KHOUS2 & -0.0144 & 0.2629 & 0.00 & 0.96 \\
\hline Year home built, $1960-74$ & KHOUS4 & -0.5684 & 0.2072 & 7.52 & 0.01 \\
\hline Year home built, $1975-79$ & KHOUS5 & -0.4774 & 0.2972 & 2.58 & 0.11 \\
\hline Year home built, $\geq 1980$ & KHOUS6 & -1.4558 & 0.3880 & 14.08 & 0.00 \\
\hline Central city location & INCITY & -0.1865 & 0.1553 & 1.44 & 0.23 \\
\hline Poor householder & POOR & -0.0865 & 0.1722 & 0.25 & 0.62 \\
\hline Hispanic origin & LATIN & 0.6047 & 0.2699 & 5.02 & 0.03 \\
\hline Black householder & RACE & 0.2312 & 0.1903 & 1.48 & 0.22 \\
\hline $\begin{array}{l}\text { Dependent variable: } \\
\text { Fuel oil/kerosine used } \\
\text { (Yes: } F O=1, \text { No: } F O=0 \text { ) }\end{array}$ & FO & & & & \\
\hline Number of observations & 1,234 & & & & \\
\hline Log likelihood & $1,640.95$ & & & & \\
\hline
\end{tabular}


TABLE 2 Ordinary-Least-Squares-Parameter Estimates for Fuel Oil/Kerosine Price Model: Northeast Census Region

\begin{tabular}{|c|c|c|c|c|c|}
\hline Variable & $\begin{array}{l}\text { Variable } \\
\text { Code }\end{array}$ & $\begin{array}{l}\text { Parameter } \\
\text { Estimate }\end{array}$ & $\begin{array}{l}\text { Standard } \\
\text { Error }\end{array}$ & $\begin{array}{c}\mathbf{T} \text { for } \mathrm{HO}: \\
\text { Parameter }=0\end{array}$ & $\begin{array}{l}\text { Frobability } \\
\text { >Absolute T }\end{array}$ \\
\hline & Intercept & 20.7439 & 0.2454 & 84.52 & 0.00 \\
\hline 1982 RECS & D1982 & 0.0857 & 0.0429 & 2.00 & 0.05 \\
\hline 1984 RECS & D1984 & 0.3309 & 0.0315 & 10.50 & 0.00 \\
\hline Cooling degree days & CDD65 & $9.8 E-5$ & $9.0 E-5$ & 1.08 & 0.28 \\
\hline Heating degree days & HDD65 & $2.02 E-5$ & $1.5 \mathrm{E}-5$ & 1.33 & 0.18 \\
\hline Consumer price index-urban & CPIU & -9.6205 & 0.1113 & -86.42 & 0.00 \\
\hline Square feet heated & HEATED & $-2.77 E-6$ & $1.5 \mathrm{E}-5$ & -0.18 & 0.86 \\
\hline Attached single-family home & SINGFAM2 & 0.1777 & 0.0882 & $\ddot{2.01}$ & 0.04 \\
\hline 2-4 Multifamily units & MULTFAM1 & -0.0502 & 0.0597 & -0.84 & 0.40 \\
\hline$>4$ Multifamily units & MULTFAM2 & -0.7305 & 0.0462 & -15.80 & 0.00 \\
\hline Mobile homes & MOBILHOM & 0.7624 & 0.1272 & 5.99 & 0.00 \\
\hline Year home built, <1940 & KHOUS1 & -0.0658 & 0.0381 & -1.73 & 0.08 \\
\hline Year home built, $1940-49$ & KHOUS2 & -0.0407 & 0.0543 & -0.75 & 0.45 \\
\hline Year home built, 1960-74 & KHOUS4 & -0.1645 & 0.0674 & -2.44 & 0.01 \\
\hline Year home built, 1975-79 & KHOUS5 & 0.0042 & 0.0766 & 0.06 & 0.96 \\
\hline Year home built, $\geq 1980$ & KHOUS6 & 0.0191 & 0.1774 & 0.11 & 0.91 \\
\hline $\begin{array}{l}\text { Central city location } \\
\text { Poor householder }\end{array}$ & $\begin{array}{l}\text { INCITY } \\
\text { POOR }\end{array}$ & $\begin{array}{r}-0.0743 \\
0.0285\end{array}$ & $\begin{array}{l}0.0373 \\
0.0338\end{array}$ & $\begin{array}{l}-2.00 \\
0.844\end{array}$ & $\begin{array}{l}0.05 \\
0.40\end{array}$ \\
\hline Hispanic origin & LATIN & -0.0557 & 0.0708 & -0.79 & 0.43 \\
\hline Black househoider & RACE & 0.0442 & 0.0466 & 0.95 & 0.34 \\
\hline Mills ratio & m & -0.1842 & 0.1342 & -1.37 & 0.17 \\
\hline \multicolumn{6}{|l|}{ Dependent variable: } \\
\hline Fuel oil/kerosine price & PRICEFK & & & & \\
\hline Number of observations & 2,569 & & & & \\
\hline Adjusted $R^{2}$ & 0.8033 & & & & \\
\hline
\end{tabular}


TABLE 3 Maximum-Likelihood-Parameter Estimates for Fuel Oil/Kerosine Fuel-Share Model: Midwest Census Region

\begin{tabular}{|c|c|c|c|c|c|}
\hline Variable & $\begin{array}{l}\text { Variable } \\
\text { Code }\end{array}$ & $\begin{array}{l}\text { Parameter } \\
\text { Estimate }\end{array}$ & $\begin{array}{c}\text { Standard } \\
\text { Error }\end{array}$ & Chi Square & Probability \\
\hline & Intercept & -0.8266 & 0.9900 & 0.70 & 0.40 \\
\hline 1981 RECS & D1981 & -0.6937 & 0.2790 & 6.18 & 0.01 \\
\hline 1982 RECS & D1982 & -1.3981 & 0.3481 & 16.13 & 0.00 \\
\hline 1984 RECS & D1984 & -0.3140 & 0.2632 & 1.42 & 0.23 \\
\hline Cooling degree days & CDD65 & $-1.24 E-4$ & 0.0011 & 0.01 & 0.91 \\
\hline Heating degree days & HDD65 & $1.7 E-4$ & $1.1 \mathrm{E}-4$ & 2.33 & .13 \\
\hline Joint degree days & CDD65*Hdd65 & $-3.299 E-7$ & $1.928 E-7$ & 2.93 & 0.09 \\
\hline Real income & INCOME & $-1.565 E-6$ & $8.442 E-6$ & 0.03 & 0.85 \\
\hline Square feet heated & HEATED & $-2.2 E-5$ & $1.2 \mathrm{E}-4$ & 0.03 & 0.86 \\
\hline Attached single-family home & SINGFAM2 & -0.9177 & 0.6319 & 2.112 & 0.15 \\
\hline 2-4 Multifamily units & MULTFAM1 & -0.7344 & 0.4012 & 3.35 & 0.67 \\
\hline$>4$ Multifamily units & MULTFAM2 & -0.9121 & 0.4520 & 4.07 & 0.04 \\
\hline Mobile homes & MOBILHOM & 0.5150 & 0.4095 & 1.58 & 0.21 \\
\hline Year home built, $<1940$ & KHOUS1 & 0.2402 & 0.3130 & 0.59 & 0.44 \\
\hline Year home built, $1940-49$ & KHOUS2 & 0.5678 & 0.3890 & 2.13 & 0.14 \\
\hline Year home built, $1960-74$ & KHOUS4 & -0.4160 & 0.3607 & 1.33 & 0.25 \\
\hline Year home built, 1975-79 & KHOUS5 & 0.1727 & 0.3920 & 0.19 & 0.66 \\
\hline Year home built, $\geq 1980$ & KHOUS6 & 0.0935 & 0.5825 & 0.03 & 0.87 \\
\hline Central city location & INCITY & -0.3587 & 0.2418 & 2.20 & 0.14 \\
\hline Poor householder & POOR & -0.4701 & 0.2921 & 2.59 & 0.11 \\
\hline Hispanic origin & LATIN & -0.4455 & 1.0442 & 0.18 & 0.67 \\
\hline Black householder & RACE & 0.2201 & 0.4690 & 0.22 & 0.64 \\
\hline $\begin{array}{l}\text { Dependent variable: } \\
\text { Fuel oil/kerosine used } \\
\text { (Yes: } F O=1 \text {, No: } F O=0 \text { ) }\end{array}$ & FO & & & & \\
\hline $\begin{array}{l}\text { Number of ohservations } \\
\text { Log likelihood }\end{array}$ & $\begin{array}{l}1,344 \\
811.77\end{array}$ & & & & \\
\hline
\end{tabular}


TABLE 4 Ordinary-Least-Squares-Parameter Estimates for Fuel Oil/Kerosine Price Model: Midwest Census Region

\begin{tabular}{|c|c|c|c|c|c|}
\hline Variable & $\begin{array}{l}\text { Variable } \\
\text { Code }\end{array}$ & $\begin{array}{c}\text { Parameter } \\
\text { Estimate }\end{array}$ & $\begin{array}{l}\text { Standard } \\
\text { Error }\end{array}$ & $\begin{array}{c}T \text { for HO: } \\
\text { Parameter }=0\end{array}$ & $\begin{array}{l}\text { Probability } \\
\text { >Absolute T }\end{array}$ \\
\hline & Intercept & 27.1144 & 2.0793 & 13.04 & 0.00 \\
\hline 1982 RECS & D1982 & 1.5310 & 0.3551 & 4.31 & 0.00 \\
\hline 1984 RECS & D1984 & 1.0987 & 0.1209 & 9.09 & 0.00 \\
\hline Cooling degree days & CDD65 & 0.0034 & 0.0007 & 4.61 & 0.00 \\
\hline Heating degree days & HDD65 & $-6.9 E-5$ & $4.8 E-5$ & -1.44 & 0.15 \\
\hline Consumer price index-urban & CPIU & -12.6786 & 1.0409 & -12.18 & 0.00 \\
\hline Square feet heated & HEATED & $-7.56 E-6$ & 5.9E-5 & -0.13 & 0.90 \\
\hline Attached singie-family home & SINGFAM2 & 1.7447 & 0.4829 & 3.61 & 0.00 \\
\hline 2-4 Multifamily units & MULTFAM1 & 0.7574 & 0.3654 & 2.07 & 0.04 \\
\hline$>4$ Multifamily units & MULTFAM2 & -0.4135 & 0.4544 & -0.910 & 0.36 \\
\hline Mobile homes & MOBILHOM & -0.3718 & 0.2716 & -1.37 & 0.17 \\
\hline Year home built, $<1940$ & KHOUS1 & -0.0717 & 0.1718 & -0.42 & 0.68 \\
\hline Year home built, $1940-49$ & KHOUS2 & -0.5529 & 0.2864 & -1.93 & 0.05 \\
\hline Year home built, 1960-74 & KHOUS4 & 0.8279 & 0.2405 & 3.44 & 0.00 \\
\hline Year home built, 1975-79 & KHOUS5 & 0.3407 & 0.2110 & 1.62 & 0.11 \\
\hline Year home built, $\geq 1980$ & KHOUS6 & 0.5847 & 0.3259 & 1.79 & 0.07 \\
\hline Central city location & INCITY & 0.6832 & 0.1967 & 3.47 & 0.00 \\
\hline Poor householder & POOR & 0.3955 & 0.2108 & 1.88 & 0.06 \\
\hline Hispanic origin & LATIN & 1.1393 & 0.3278 & 3.48 & 0.00 \\
\hline Black householder & RACE & 0.0577 & 0.2672 & 0.22 & 0.83 \\
\hline Mills ratio & $m$ & 1.5088 & 0.4081 & 3.70 & 0.00 \\
\hline Dependent variable: & & & & & \\
\hline Fuel oil/kerosine price & PRICEFK & & & & \\
\hline Number of observations & 601 & & & & \\
\hline Adjusted $R^{2}$ & 0.4049 & & & & \\
\hline
\end{tabular}


TABLE 5 Maximum-Likelihood-Parameter Estimates for Fuel Oil/Kerosine Fuel-Share Model: South Census Region

\begin{tabular}{|c|c|c|c|c|c|}
\hline Variable & $\begin{array}{l}\text { Variable } \\
\text { Code }\end{array}$ & $\begin{array}{c}\text { Parameter } \\
\text { Estimate }\end{array}$ & $\begin{array}{l}\text { Standard } \\
\text { Error }\end{array}$ & Chi Square & Frobability \\
\hline & Intercept & -1.0062 & 0.9921 & 1.03 & 0.32 \\
\hline 1981 RECS & D1981 & -1.1050 & 0.2601 & 18.05 & 0.00 \\
\hline 1982 RECS & 01982 & -0.9662 & 0.2932 & 10.86 & 0.00 \\
\hline 1984 RECS & 01984 & -0.3399 & 0.2479 & 1.88 & 0.17 \\
\hline Cooling degree days & CDD65 & $-8.9 E-5$ & 2.7E-4 & 0.11 & 0.74 \\
\hline Heating degree days & HDD65 & 3.7E-4 & $1.6 \mathrm{E}-4$ & 5.44 & 0.02 \\
\hline Joint degree days & CDD65*Hdd65 & $-2.5 E-7$ & $7.4 E-8$ & 11.03 & 0.00 \\
\hline Real income & NCONE & $-2.5 E-6$ & 8.6E-6 & 0.08 & 0.77 \\
\hline Square feet heated & HEATED & $1.2 E-4$ & $1.4 E-4$ & 0.70 & 0.40 \\
\hline 2-4 Multifamily units & MULTFAM1 & -0.2111 & 0.3882 & 0.30 & 0.59 \\
\hline$>4$ Multifamily units & MULTFAM2 & -0.7117 & 0.4398 & 2.62 & 0.11 \\
\hline Mobile homes & MOBILHOM & 1.2525 & 0.3108 & 16.24 & $0.0 n$ \\
\hline Year home built, $<1940$ & KHOUS1 & 0.1611 & 0.2896 & 0.31 & 0.58 \\
\hline Year home built, $1940-49$ & KHOUS2 & -0.5389 & 0.3692 & 2.13 & 0.14 \\
\hline Year home built, 1960-74 & KHOUS4 & -0.4021 & 0.2705 & 2.21 & 0.14 \\
\hline Year home built, 1975-79 & KHOU:55 & -0.4636 & 0.3525 & 1.73 & 0.19 \\
\hline Year home built, $\geq 1980$ & KHOU $\$ 6$ & -1.4875 & 0.5055 & 8.66 & 0.00 \\
\hline Central city location & INCITY & -0.2031 & 0.2218 & 0.84 & 0.36 \\
\hline Poor householder & POOR' & $2.4 E-4$ & 0.2363 & 0.00 & 0.99 \\
\hline Black householder & RACE & 0.0079 & 0.2359 & 0.00 & 0.97 \\
\hline $\begin{array}{l}\text { Dependent variable: } \\
\text { Fuel oil/kerosine used } \\
\text { (Yes: } F O=1, \text { No: } \quad F O=0 \text { ) }\end{array}$ & FO & & & & \\
\hline Number of observations & 1,173 & & & & \\
\hline Log likelihood & 844.96 & & & & \\
\hline
\end{tabular}


Table 6 shows the OLS parameter estimates for the South census region fuel oil/kerosine price model. Several variables are significani at the 5\% level: the CPIU, larger-than-four multifamily homes, the 1984 RECS dummy variable, homes built after 1959 , homes built before 1940 , and black households.

\subsubsection{West Census Region}

Table 7 shows the West census region maximum-likelihood-parameter estimates for the fuel oil/kerosine fuel-share model. Three variables are significant at the 5\% level: square feet heated, cooling degree days, and real income.

Table 8 shows the OLS-parameter estimates for the West census region fuel oil/kerosine price model. Seven variables are significant at the 5\% level: the CPIU, homes built after 1979, the 1984 RECS dummy variable, homes built from 1975 through 1979, mobile homes, mills ratio, and the 1982 RECS dummy variable.

\subsection{Natural Gas Share and Price Models}

\subsubsection{Northeast Census Region}

Table 9 shows the Northeast census region maximum-likelihood-parameter-estimates for the natural gas fuel-share model. The following variables are statistically significant at the 5\% level: central city location, homes built from 1975 through 1979, the black household dummy variable, heating degree days, two-to-four multifamily units, homes built from 1940 through 1949 , the 1982 RECS dummy variable, the 1981 RECS dummy variable, joint degree days, homes built before 1940, real income, larger-than-four multifamily units, and square feet heated.

Table 10 shows the OLS-parameter estimates for the Northeast census region natural gas price model. Ten variables are significant at the 5\% level: larger-than-four multifamily units, the 1984 RECS dummy variable, cooling degree days, central city location, the CPIU, two-to-four multifamily units, homes built from 1960 through 1974, homes built before 1940, 1982 RECS dummy variable, and heating degree days.

\subsubsection{Midwest Census Region}

Table 11 shows the Midwest census region maximum-likelihood-parameter estimates for the natural gas fuel-share model . Variables significant at the 5\% level are homes built after 1959 , central city location, joint degree days, two-to-four multifamily units, cooling degree days, black households, large multifamily units, homes built before 1940, and heating degree days. 
TABLE 6 Ordinary-Least-Squares-Parameter Estimates for Fuel Oil/Kerosine Price Model: South Census Region

\begin{tabular}{|c|c|c|c|c|c|}
\hline Variable & $\begin{array}{l}\text { Variable } \\
\text { Code }\end{array}$ & $\begin{array}{l}\text { Parameter } \\
\text { Estimate }\end{array}$ & $\begin{array}{l}\text { Standard } \\
\text { Error }\end{array}$ & $\begin{array}{c}\text { T for } \mathrm{HO}: \\
\text { Parameter }=0\end{array}$ & $\begin{array}{l}\text { Probability } \\
\text { >Absolute T }\end{array}$ \\
\hline & Intercept & 17.5536 & 1.2312 & 14.26 & 0.00 \\
\hline 1982 RECS & D1982 & 0.1120 & 0.1254 & 0.89 & 0.37 \\
\hline 1984 RECS & D1984 & 0.5759 & 0.1011 & 5.70 & 0.00 \\
\hline Cooling degree days & CDD65 & $1.3 E-4$ & $1.5 E-4$ & 0.87 & 0.38 \\
\hline Heating degree days & HDD65 & $-8.5 E-5$ & $7.1 E-5$ & -1.20 & 0.23 \\
\hline Consumer price index-urban & CPIU & -6.7205 & 0.6054 & -11.10 & 0.00 \\
\hline Square feet heated & HEATED & $-8.9 E-5$ & $6.3 E-5$ & -1.41 & 0.16 \\
\hline Attached single-family home & SINGFAM2 & 1.1503 & 0.6117 & 1.88 & 0.06 \\
\hline 2-4 Multifamily units & MULTFAM1 & -0.0094 & 0.2022 & -0.05 & 0.96 \\
\hline$>4$ Multifamily units & MULTFAM2 & -1.4361 & 0.2207 & -6.510 & 0.00 \\
\hline Mobile homes & MOBILHOM & -0.3726 & 0.2293 & -1.62 & 0.10 \\
\hline Year home built, <1940 & KHOUS1 & 0.3168 & 0.1284 & 2.47 & 0.01 \\
\hline Year home built, $1940-49$ & KHOUS2 & 0.1107 & 0.1698 & 0.65 & 0.51 \\
\hline Year home built, $1960-74$ & KHOUS4 & 0.3923 & 0.1405 & 2.79 & 0.01 \\
\hline Year home built, $1975-79$ & KHOUS5 & 0.7130 & 0.1834 & 3.89 & 0.00 \\
\hline Year home built, $\geq 1980$ & KHOUS6 & 0.8987 & 0.3570 & 2.52 & 0.01 \\
\hline Central city location & INCITY & 0.0237 & 0.1149 & 0.21 & 0.84 \\
\hline Foor householder & POOA & -0.0293 & 0.0952 & -0.31 & 0.76 \\
\hline Hispanic origin & LATIN & -0.0319 & 0.3441 & -0.09 & 0.93 \\
\hline Black householder & RACE & 0.2052 & 0.1045 & 1.96 & 0.05 \\
\hline Mills ratio & m & 0.0240 & 0.1738 & 0.14 & 0.89 \\
\hline \multicolumn{6}{|l|}{ Dependent variable: } \\
\hline Fuel oil/kerosine price & PRICEFK & & & & \\
\hline Number of observations & 911 & & & & \\
\hline Adjusted $\mathrm{R}^{2}$ & 0.3174 & & & & \\
\hline
\end{tabular}


TABLE 7 Maximum-Likelihood-Parameter Estimates for Fuel Oil/Kerosine Fuel-Share Model: West Census Region

\begin{tabular}{|c|c|c|c|c|c|}
\hline Variable & $\begin{array}{l}\text { Variable } \\
\text { Code }\end{array}$ & $\begin{array}{c}\text { Parameter } \\
\text { Estimate }\end{array}$ & $\begin{array}{l}\text { Standard } \\
\text { Error }\end{array}$ & Chi Square & Probability \\
\hline & Intercept & -1.9206 & 0.9740 & 3.89 & 0.05 \\
\hline 1981 RECS & D1981 & 0.1470 & 0.4873 & 0.09 & 0.76 \\
\hline 1982 RECS & Di982 & -0.2147 & 0.5139 & 0.17 & 0.68 \\
\hline 1984 RECS & 01984 & 0.4378 & 0.5060 & 0.75 & 0.39 \\
\hline Cooling degree days & CDD65 & -0.0022 & 0.0011 & 4.23 & 0.04 \\
\hline Heating degree days & HDD65 & $-1.8 E-4$ & $1.3 E-4$ & 1.84 & 0.17 \\
\hline Joint degree days & CDD65*Hdd65 & $3.0 E-7$ & $2.4 E-7$ & 1.53 & 0.22 \\
\hline Real income & INCONE & $-3.8 E-5$ & $1.8 E-5$ & 4.23 & 0.04 \\
\hline Square feet heated & HEATED & 7.7E-4 & $1.9 E-4$ & 16.46 & 0.00 \\
\hline Mobile homes & MOBILHOM & -0.5531 & 1.0732 & 0.27 & 0.61 \\
\hline Year home built, $<1940$ & KHOUS1 & 0.8347 & 0.4639 & 3.24 & 0.07 \\
\hline Year home built, $1940-49$ & KHOUS2 & 0.5609 & 0.5936 & 0.89 & 0.35 \\
\hline Year home built, $1960-74$ & KHOUS4 & -0.6196 & 0.5330 & 1.35 & 0.25 \\
\hline Year home built, 1975--79 & KHOUS5 & -0.8698 & 0.7134 & 1.49 & 0.22 \\
\hline Year home built, $\geq 1980$ & KHOUS6 & -1.1832 & 1.1062 & 1.14 & 0.28 \\
\hline Central city location & INCITY & -0.6291 & 0.3806 & 2.73 & 0.10 \\
\hline Poor householder & POOR & -1.0906 & 0.5940 & 3.37 & 0.07 \\
\hline Black householder & RACE & -0.7889 & 1.0534 & 0.56 & 0.45 \\
\hline $\begin{array}{l}\text { Dependent variable: } \\
\text { Fuel oil/kerosine used } \\
\text { (Yes: } F O=1 \text {, No: } F O=0 \text { ) }\end{array}$ & FO & & & & \\
\hline $\begin{array}{l}\text { Number of observations } \\
\text { Log likelihood }\end{array}$ & $\begin{array}{l}1,035 \\
304.88\end{array}$ & & & & \\
\hline
\end{tabular}


TABLE 8 Ordinary-Least-Squares-Parameter Estimates for Fuel Oil/Kerosine Price Model: West Census Region

\begin{tabular}{|c|c|c|c|c|c|}
\hline Variable & $\begin{array}{l}\text { Variable } \\
\text { Code }\end{array}$ & $\begin{array}{l}\text { Parameter } \\
\text { Estimate }\end{array}$ & $\begin{array}{l}\text { Standard } \\
\text { Error }\end{array}$ & $\begin{array}{c}\mathrm{T} \text { for } \mathrm{HO}: \\
\text { Parameter }=0\end{array}$ & $\begin{array}{l}\text { Probability } \\
\text { >Absolute T }\end{array}$ \\
\hline & Intercept & 18.4287 & 1.2815 & 14.38 & 0.00 \\
\hline 1982 RECS & D1982 & 0.3850 & 0.1615 & 2.38 & 0.02 \\
\hline 1984 RECS & D1984 & 0.7755 & 0.1815 & 4.27 & $0.0 \mathrm{C}$ \\
\hline Cooling degree days & CDD65 & $1.5 \mathrm{E}-4$ & $2.4 E-4$ & 0.62 & 0.54 \\
\hline Heating degree days & HDD65 & $-6.3 E-5$ & 5.4E-5 & -1.18 & 0.24 \\
\hline Consumer price index-urban & CPIU & -8.4142 & 0.7939 & -10.60 & 0.00 \\
\hline Square feet heated & HEATED & $1.4 \mathrm{E}-4$ & $8.9 E-5$ & 1.59 & 0.11 \\
\hline Attached single-family home & SINGFAM2 & 0.8027 & 0.9819 & 0.82 & 0.41 \\
\hline 2-4 Multifamily units & MULTFAM1 & -0.3945 & 0.4157 & -0.95 & 0.34 \\
\hline$>4$ Multifamily units & MULTFAM2 & 0.7298 & 0.6959 & 1.05 & 0.30 \\
\hline Mobile homes & MOBILHOM & -1.3084 & 0.4348 & -3.01 & 0.00 \\
\hline Year home built, <1940 & KHOOUS1 & 0.0944 & 0.2013 & 0.47 & 0.64 \\
\hline Year home built, $1940-49$ & KHOUS2 & -0.0154 & 0.2297 & -0.07 & 0.95 \\
\hline Year home built, 1960-74 & KHOUS4 & -0.132 .8 & 0.1948 & -0.68 & 0.50 \\
\hline Year home built, 1975-79 & KHONS5 & 1.5745 & 0.3800 & 4.14 & 0.00 \\
\hline Year home built, $\geq 1980$ & KHOUS6 & 4.6254 & 0.5319 & 8.70 & 0.00 \\
\hline Central city location & INYCITY & -0.0724 & 0.1651 & -0.44 & 0.66 \\
\hline Poor householder & POOR & -0.3311 & 0.1987 & -1.67 & 0.10 \\
\hline Hispanic origin & LATIN & 0.6920 & 0.3572 & 1.94 & 0.05 \\
\hline Black householder & RACE & 0.7940 & 0.5868 & 1.35 & 0.18 \\
\hline Mills ratio & $m$ & -0.2925 & 0.1080 & -2.71 & 0.01 \\
\hline
\end{tabular}

Dependent variable:

Fuel oil/kerosine price Number of observations PRICEFK Adjusted $\mathrm{R}^{2}$ 
TABLE $\&$ Maximum-Likelihood-Parameter Estimates for Natural Gas Fuel-Share Model: Northeast Census Region

\begin{tabular}{|c|c|c|c|c|c|}
\hline Variable & $\begin{array}{l}\text { Variable } \\
\text { Code }\end{array}$ & $\begin{array}{c}\text { Parameter } \\
\text { Estimate }\end{array}$ & $\begin{array}{l}\text { Standard } \\
\text { Error }\end{array}$ & Chi Square & Probability \\
\hline & Intercept & 0.1247 & 0.9690 & 0.02 & 0.90 \\
\hline 1981 RECS & D1994 & 0.6302 & 0.2065 & 9.31 & 0.00 \\
\hline 1982 RECS & D1982 & 0.7567 & 0.2478 & 9.33 & 0.00 \\
\hline 1984 RECS & D1984 & 0.3281 & 0.2052 & 2.56 & 0.11 \\
\hline Cooling degree deys & CDD65 & -0.0020 & 0.0013 & 2.47 & 0.12 \\
\hline Heating degree days & HDD65 & $-4.5 E-4$ & $1.3 E-4$ & 12.54 & 0.00 \\
\hline Joint degree days & CDD65*Hdd65 & $6.4 \mathrm{E}-7$ & 2.3E-7 & 7.74 & 0.01 \\
\hline Real income & INCOME & $1.0 \mathrm{E}-5$ & $5.2 E-6$ & 5.01 & 0.03 \\
\hline Square feet heated & HEATED & $1.9 \mathrm{E}-4$ & 9. 1E-5 & 4.29 & 0.04 \\
\hline Attached single-family home & SINGFAM2 & 0.5130 & 0.3189 & 2.59 & 0.11 \\
\hline $2 \rightarrow$ Multifanrily units & MULTFAM1 & 0.7873 & 0.2257 & 12.16 & 0.00 \\
\hline$>4$ Multifamily units & MULTFAM2 & 0.5392 & 0.2487 & 4.70 & 0.03 \\
\hline Mobile homes & MOBILHOM & -0.4088 & 0.5426 & 0.57 & 0.45 \\
\hline Year home built, <1940 & KHOUS1 & 0.5420 & 0.2186 & 6.15 & 0.01 \\
\hline Year home built, $1940-49$ & KHCUS2 & 0.9665 & 0.3118 & $9.6 i$ & 0.00 \\
\hline Year home built, 1960-74 & KHOUS4 & 0.1969 & 0.2295 & 0.74 & 0.39 \\
\hline Year home built, 1975-79 & KHOUS5 & -2.1470 & 0.4847 & 19.62 & 0.00 \\
\hline Year home built, $\geq 1980$ & KHOUS6 & -0.6448 & 0.4165 & 2.40 & 0.12 \\
\hline Central city location & INCITY. & 1.3819 & 0.1888 & 53.58 & 0.00 \\
\hline Poor householder & POCA & 0.2092 & 0.2130 & 0.96 & 0.33 \\
\hline Hispanic origin & LATIN & 0.3436 & 0.3489 & 0.97 & 0.32 \\
\hline Black householder & RACE & 1.1416 & 0.3132 & 13.29 & 0.00 \\
\hline $\begin{array}{l}\text { Dependent variable: } \\
\text { Natural gas used } \\
\text { (Yes: } N G=1 \text {, No: } N G=0 \text { ) }\end{array}$ & NG & & & & \\
\hline $\begin{array}{l}\text { Number of observations } \\
\text { Log likelihood }\end{array}$ & $\begin{array}{l}1,234 \\
1,230.42\end{array}$ & & & & \\
\hline
\end{tabular}


TABLE 10 Drdinary-Least-Squares-Parameter Estimates for Natural Gas Price Model: Northeast Census Region

\begin{tabular}{|c|c|c|c|c|c|}
\hline Variable & $\begin{array}{l}\text { Variable } \\
\text { Code }\end{array}$ & $\begin{array}{l}\text { Parameter } \\
\text { Estimate }\end{array}$ & $\begin{array}{l}\text { Standard } \\
\text { Error }\end{array}$ & $\begin{array}{c}T \text { for HO: } \\
\text { Parameter }=0\end{array}$ & $\begin{array}{l}\text { Probability } \\
\text { >Absolute } T\end{array}$ \\
\hline & Intercept & 11.2782 & 2.5566 & 4.41 & 0.00 \\
\hline 1982 RECS & D1982 & 1.2288 & 0.5062 & 2.43 & 0.02 \\
\hline 1984 RECS & D1984 & 1.8836 & 0.3738 & 5.04 & 0.00 \\
\hline Cooling degree days & CDD65 & 0.0045 & 0.0010 & 4.35 & 0.00 \\
\hline Heating degree days & HDD65 & $-4.9 E-4$ & $2.1 \mathrm{E}-4$ & -2.30 & 0.02 \\
\hline Consumer price index-urban & CPIU & -4.5342 & 1.5604 & -2.91 & 0.00 \\
\hline Square feet heated & HEATED & 1.3E-5 & 2.1E-4 & 0.06 & 0.95 \\
\hline Attached single-family home & SINGFAM2 & -0.6242 & 0.6171 & -1.01 & 0.31 \\
\hline 2-4 Multifamily units & MULTFAM1 & 1.4038 & 0.5586 & 2.514 & 0.01 \\
\hline$>4$ Multifamily units & MULTFAM2 & 3.2489 & 0.5787 & 5.61 & 0.00 \\
\hline Mobile homes & MOBHLHOM & -0.6230 & 1.5231 & -0.41 & 0.68 \\
\hline Year home built, <1940 & KHOUS1 & 1.2969 & 0.5452 & 2.38 & 0.02 \\
\hline Year home built, 1940-49 & KHOUS2 & 0.6494 & 0.7539 & 0.86 & 0.39 \\
\hline Year home built, $1960-74$ & KHOUS4 & -1.4253 & 0.5779 & -2.47 & 0.01 \\
\hline Year home built, 1975-79 & KHOUS5 & -2.8579 & 1.8045 & -1.58 & 0.11 \\
\hline Year home built, $\geq 1980$ & KHOUS6 & -1.058 & 1.3107 & .0 .81 & 0.42 \\
\hline Central city location & INCITY & 1.8960 & 0.6205 & 3.06 & 0.00 \\
\hline Poor householder & POOA & -0.3118 & 0.3894 & -0.80 & 0.42 \\
\hline Hispanic origin & LATIN & 0.2393 & 0.5818 & 0.41 & 0.68 \\
\hline Black householder & RACE & -0.7399 & 0.4817 & -1.54 & 0.12 \\
\hline Mills ratio & $m$ & -1.0129 & 0.8068 & -1.26 & 0.21 \\
\hline $\begin{array}{l}\text { Dependent variable: } \\
\text { Natural gas price }\end{array}$ & PRICENG & & & & \\
\hline $\begin{array}{l}\text { Number of observations } \\
\text { Adiusted } R^{2}\end{array}$ & 2,901 & & & & \\
\hline & 0.0765 & & & & \\
\hline
\end{tabular}


TABLE 11 Maximum-Likglihood-Parameter Estimates for Natural Gas Fuel-Share Model: Midwest Census Region

\begin{tabular}{|c|c|c|c|c|c|}
\hline Variable & $\begin{array}{l}\text { Variable } \\
\text { Code }\end{array}$ & $\begin{array}{l}\text { Parameter } \\
\text { Estimate }\end{array}$ & $\begin{array}{l}\text { Standard } \\
\text { Error }\end{array}$ & Chi Square & Probability \\
\hline & Intercept & 1.6704 & 0.7175 & 5.42 & 0.02 \\
\hline 1981 RECS & D1981 & -0.3440 & 0.1922 & 3.20 & 0.07 \\
\hline 1982 RECS & D1982 & 0.1355 & 0.2216 & 0.37 & 0.54 \\
\hline 1984 RECS & D1984 & 0.0527 & 0.1969 & 0.07 & 0.79 \\
\hline Cooling degree days & CDD65 & -0.0020 & $6.6 \mathrm{E}-4$ & 9.15 & 0.00 \\
\hline Heating degree days & HDD65 & $-j .6 \mathrm{E}-4$ & 8.2E-5 & 3.77 & 0.05 \\
\hline Joint degree days & CDD65*Hdd65 & $4.6 E-7$ & $1.2 E-7$ & 13.99 & 0.00 \\
\hline Real income & INCOME & $8.2 E-6$ & $6.2 E-6$ & 1.71 & 0.19 \\
\hline Square feet heated & HEATED & 8.0E-5 & $9.1 E-5$ & 0.72 & 0.40 \\
\hline Attached single-family home & SINGFAM2 & 0.0950 & 0.3485 & 0.072 & 0.79 \\
\hline 2-4 Multifamily units & MULTFAM1 & 1.0680 & 0.3344 & 10.20 & 0.00 \\
\hline$>4$ Multifamily units & MULTFAM2 & 0.6748 & 0.2672 & 6.38 & $0.0 i$ \\
\hline Mobile homes & MOBILHOM & -0.2893 & 0.2914 & 0.99 & 0.32 \\
\hline Year home built, $<1940$ & KHOUS1 & -0.5163 & 0.2518 & 4.21 & 0.04 \\
\hline Year home built, $1940-49$ & KHOUS2 & -0.3946 & 0.3376 & 1.37 & 0.24 \\
\hline Year home built, 1960-74 & KHOUS4 & -0.8856 & 0.2604 & 11.56 & 0.00 \\
\hline Year home built, 1975-79 & KHOUS5 & -1.4682 & 0.2884 & 25.92 & 0.00 \\
\hline Year home built, $\geq 1980$ & KHOUS6 & -1.8587 & 0.3959 & 22.04 & 0.00 \\
\hline Central city location & INCITY & 0.7396 & 0.1759 & 17.67 & 0.00 \\
\hline Poor householder & POOR & -0.2229 & 0.1974 & 1.28 & 0.26 \\
\hline Hispanic origin & LATIN & 1.3711 & 0.7753 & 3.13 & 0.08 \\
\hline Black householder & RACE & 2.1046 & 0.7359 & 8.18 & 0.00 \\
\hline $\begin{array}{l}\text { Dependent variable: } \\
\text { Natural gas used } \\
\text { (Yes: NG=1, No: } \quad N G=0 \text { ) }\end{array}$ & NG & & & & \\
\hline Number of observations & 1,344 & & & & \\
\hline Log likelihood & $1,367.15$ & & & & \\
\hline
\end{tabular}


Table 12 shows the OLS-parameter estimates for the Midwest census region natural gas fuel-price model. The following variables are significant at the 5\% level: 1984 RECS dummy variable, 1982 RECS dummy variable, the CPIU, cooling degree days, central city location, twoto-four multifamily units, homes built after 1959, homes built before 1940, larger than four multifamily units, mills ratio, heating degree days, and Hispanic ethnicity.

\subsubsection{Suth Census Region}

Table 13 shows the South census region maximum-likelihood-parameter estimates for the natural gas fuel-share model. Variables significant at the 5\% level are central city location, joint degree days, homes built after 1974, the 1982 RECS dummy variable, black households, mobile homes, Hispanic ethnicity, and two-to-four multifamily units.

Table 14 shows the OLS-parameter estimates for the South census region natural gas price model. All variables except heating degree days and homes built in the 1960-74 period are significant at the $5 \%$ level.

\subsubsection{West Census Region}

Table 15 shows the West census region maximum-likelihood-parameter estimates for the natural gas fuel-share model. Variables significant at the $5 \%$ level are homes built after 1959, joint degree days, Hispanic ethnicity, cooling degree days, central city location, heating degree days, real income, homes built from 1940 through 1949, the 1981 RECS dummy variable, mobile homes, and homes built before 1940 .

Table 16 shows the OLS-parameter estimates for the We it census region natural gas price model. Six variables are significant at the 5\% level: the 1984 'RECS dummy variable, the 1982 RECS dummy variable, cocling degree days, homes built betwe 2 n 1940 and 1949, the CPIU, and heating degree days.

\subsection{Liquefied Petroleum Gas Share and Price Models}

\subsubsection{Northeast Census Region}

Table 17 shows the Northeast census region maximum-likelihood-parameter estimates for the LPG fuel-share model. Variables significant at the 5\% level are mobile homes, central city location, real income, and poor households. 
TABLE 12 Ordinary-Least-Squares-Parameter Estimates for Natural Gas Price Model: Midwest Census Region

\begin{tabular}{|c|c|c|c|c|c|}
\hline Variable & $\begin{array}{l}\text { Variable } \\
\text { Code }\end{array}$ & $\begin{array}{c}\text { Parameter } \\
\text { Estimate }\end{array}$ & $\begin{array}{l}\text { Standard } \\
\text { Error }\end{array}$ & $\begin{array}{c}T \text { for } \mathrm{HO}: \\
\text { Parameter }=0\end{array}$ & $\begin{array}{l}\text { Probability } \\
\text { >Absolute T }\end{array}$ \\
\hline & Intercept & 0.2788 & 0.3854 & 0.72 & 0.46 \\
\hline 1982 RECS & D1982 & 0.5765 & 0.0517 & $11 . i 6$ & 0.00 \\
\hline 1984 RECS & D1984 & 0.8404 & 0.0438 & 19.18 & 0.00 \\
\hline Cooling degree days & CDD65 & $-9.9 \mathrm{E}-4$ & $7.3 E-5$ & -13.48 & 0.00 \\
\hline Heating degree days & HDD65 & $-9.4 E-5$ & $1.8 \mathrm{E}-5$ & -5.18 & 0.00 \\
\hline Consumer price index-urban & CPIU & 3.9566 & 0.2065 & 19.16 & 0.00 \\
\hline Square feet heated & HEATED & $-4.7 E-6$ & $2.2 E-5$ & -0.22 & 0.83 \\
\hline Attached single-family home & SINGFAM2 & 0.1664 & 0.1007 & 1.65 & 0.10 \\
\hline 2-4 Multifamily units & MULTFAM1 & 0.4136 & 0.0673 & 6.14 & 0.00 \\
\hline$>4$ Multifamily units & MULTFAM2 & 0.3759 & 0.0709 & 5.31 & 0.00 \\
\hline Mobile homes & MOBILHOM & -0.0296 & 0.1071 & -0.28 & 0.78 \\
\hline Year home built, $<1940$ & KHOUS1 & -0.3283 & 0.0575 & $-5.7 i$ & 0.00 \\
\hline Year home built, $1940-49$ & KHOUS2 & -0.1110 & 0.0747 & -1.49 & 0.14 \\
\hline Year home built, 1960-74 & KHOUS4 & -0.2145 & 0.0697 & -3.08 & 0.00 \\
\hline Year home built, 1975-79 & KHOUSS & -0.3453 & 0.1097 & -3.15 & 0.00 \\
\hline Year home built, $\geq 1980$ & KHOUS6 & -0.4543 & 0.1529 & -2.97 & 0.00 \\
\hline Central city location & INCITY & 0.3587 & 0.0514 & 6.98 & 0.00 \\
\hline Poor householder & POOR & -0.0573 & 0.0481 & -1.19 & 0.23 \\
\hline Hispanic origin & LATIN & 0.3022 & 0.1205 & 2.51 & 0.01 \\
\hline Black householder & RACE & 0.1078 & 0.0785 & 1.37 & 0.17 \\
\hline Mills ratio & $\mathbf{m}$ & -0.6443 & 0.1224 & -5.27 & 0.00 \\
\hline $\begin{array}{l}\text { Dependent variable: } \\
\text { Natural gas price }\end{array}$ & PRICENG & & & & \\
\hline Number of observations & 4,031 & & & & \\
\hline Adjusted $\mathrm{R}^{2}$ & 0.2209 & & & & \\
\hline
\end{tabular}


TABLE 13 Maximum-Likelihood-Parameter Estimates for Natural Gas Fuel-Share Model: South Census Region

\begin{tabular}{|c|c|c|c|c|c|}
\hline Variable & $\begin{array}{l}\text { Variable } \\
\text { Code }\end{array}$ & $\begin{array}{c}\text { Parameter } \\
\text { Estimate }\end{array}$ & $\begin{array}{l}\text { Standard } \\
\text { Error }\end{array}$ & Chi Square & Probability \\
\hline & Intercept & -2.3331 & 0.7428 & 9.87 & 0.00 \\
\hline 1981 RECS & D1981 & 0.3384 & 0.1875 & 3.26 & 0.07 \\
\hline 1982 RECS & D1982 & 0.7856 & 0.2167 & 13.14 & 0.00 \\
\hline 1984 AECS & 01984 & 0.2232 & 0.1979 & 1.27 & 0.26 \\
\hline Cooling degree days & CDD65 & $1.4 \mathrm{E}-4$ & $1.9 \mathrm{E}-4$ & 0.49 & 0.49 \\
\hline Heating degree days & HDD65 & $-5.7 E-5$ & $1.2 E-4$ & 0.21 & 0.65 \\
\hline Joint degree days & CDD65*Hdd 65 & $3.2 E-7$ & 5.7E-8 & 32.67 & 0.00 \\
\hline Real income & INCONE & $8.3 E-6$ & $6.6 E-6$ & 1.60 & 0.21 \\
\hline Square feet heated & HEATED & $1.3 E-4$ & $1.1 E-4$ & 1.38 & 0.24 \\
\hline Attached single-family home & SINGFAM2 & 0.1909 & 0.4260 & 0.20 & 0.65 \\
\hline 2-4 Multifamily units & MULTFAM1 & 0.5106 & 0.2617 & 3.81 & 0.05 \\
\hline$>4$ Multifamily units & MULTFAM2 & -0.1961 & 0.2519 & 0.61 & 0.44 \\
\hline Mobile homes & MOBILHOM & -1.0415 & 0.3222 & 10.45 & 0.00 \\
\hline Year home built, $<1940$ & KHOUS1 & 0.0743 & 0.2315 & 0.10 & 0.75 \\
\hline Year home built, $1940-49$ & KHOUS2 & 0.4158 & 0.2625 & 2.51 & 0.11 \\
\hline Year home built, 1960-74 & KHOUS4 & -0.0080 & 0.2010 & 0.00 & 0.97 \\
\hline Year home built, 1975-79 & KHOUS5 & -1.3456 & 0.2771 & 23.59 & 0.00 \\
\hline Year home built, $\geq 1980$ & KHOUS6 & -1.8031 & 0.3435 & 27.56 & 0.00 \\
\hline Central city location & INCITY & 1.1211 & 0.1627 & 47.50 & 0.00 \\
\hline Poor householder & POOR & -0.0351 & 0.1783 & 0.04 & 0.84 \\
\hline Hispanic origin & LATIN & 1.2772 & 0.4456 & 8.21 & 0.00 \\
\hline Black householder & RACE & 0.5774 & 0.1696 & 11.58 & 0.00 \\
\hline $\begin{array}{l}\text { Dependent variable: } \\
\text { Natural gas used } \\
\text { (Yes: } N G=1 \text {, No: } N G=0 \text { ) }\end{array}$ & NG & & & & \\
\hline Number of observations & 1,173 & & & & \\
\hline Log likelihood & $1,325.19$ & & & & \\
\hline
\end{tabular}


TABLE 14 Ordinary-Least-Squares-Parameter Estimates for Natural Gas Price Model: South Census Fiegion

\begin{tabular}{|c|c|c|c|c|c|}
\hline Variahle & $\begin{array}{l}\text { Variable } \\
\text { Code }\end{array}$ & $\begin{array}{l}\text { Parameter } \\
\text { Estimate }\end{array}$ & $\begin{array}{l}\text { Standard } \\
\text { Error }\end{array}$ & $\begin{array}{c}T \text { for } \mathrm{HO}: \\
\text { Parameter }=0\end{array}$ & $\begin{array}{l}\text { Probability } \\
\text { >Absolute T }\end{array}$ \\
\hline & Intercept & -2.8111 & 0.6010 & -4.68 & 0.00 \\
\hline 1982 RECS & D1982 & 1.3465 & 0.0936 & 14.39 & 0.00 \\
\hline 1984 RECS & D1984 & 0.5776 & 0.07809 & 7.40 & 0.00 \\
\hline Cooling degree days & CDD65 & $6.8 E-4$ & $1.0 \mathrm{E}-4$ & 6.63 & 0.00 \\
\hline Heating degree days & HDD65 & 4.1E-5 & $5.7 E-5$ & 0.72 & 0.47 \\
\hline Consumer price index-urban & CPIU & 2.6270 & 0.3051 & 8.61 & 0.00 \\
\hline Square feet heated & HEATED & $1.2 E-4$ & 5.0E-5 & 2.50 & 0.01 \\
\hline Attached single-family home & SINGFAM2 & 1.0080 & 0.2056 & 4.90 & 0.00 \\
\hline 2-4 Multifamily units & MULTFAM1 & 0.9162 & 0.1162 & 7.89 & 0.00 \\
\hline$>4$ Multifamily units & MULTFAM2 & 0.3953 & 0.1174 & 3.37 & 0.00 \\
\hline Mobile homes & MOBILHOM & -1.2961 & 0.2467 & -5.25 & 0.00 \\
\hline Year home built, <1940 & KHOUST & 0.4185 & 0.0992 & 4.22 & 0.00 \\
\hline Year home built, 1940-49 & KHOUS2 & 0.9917 & 0.1168 & 8.49 & 0.00 \\
\hline Year home built, 1960-74 & KHOUS4 & 0.1109 & 0.0904 & 1.23 & 0.22 \\
\hline Year home built, 1975-79 & KHOUS5 & -2.0172 & 0.1978 & -10.20 & 0.00 \\
\hline Year home built, $\geq 1980$ & KHOUS6 & -2.5456 & 0.2861 & -8.90 & 0.00 \\
\hline Central City Location & INCITY & 0.7286 & 0.1116 & 6.53 & 0.00 \\
\hline Poor Householder & POOR & -0.3026 & 0.0772 & -3.92 & 0.00 \\
\hline Hispanic Origin & LATIN & 0.7949 & 0.1629 & 4.88 & 0.00 \\
\hline Black Householder & RACE & 0.8373 & 0.0897 & 9.34 & 0.00 \\
\hline Mills Ratio & $\mathrm{m}$ & -1.9578 & 0.1441 & -13.59 & 0.00 \\
\hline $\begin{array}{l}\text { Dependent variable: } \\
\text { Natural gas price }\end{array}$ & PRICENG & & & & \\
\hline Number of observations & 3,735 & & & & \\
\hline Adjusted $R^{2}$ & 0.1555 & & & & \\
\hline
\end{tabular}


TABLE 15 Maximum-Likelifijod-Parameter Estimates for Natural Gas Fuel-Share Model: West Census Region

\begin{tabular}{|c|c|c|c|c|c|}
\hline Variable & $\begin{array}{l}\text { Variable } \\
\text { Code }\end{array}$ & $\begin{array}{l}\text { Parameter } \\
\text { Estimate }\end{array}$ & $\begin{array}{l}\text { Standard } \\
\text { Error }\end{array}$ & Chi Square & Probability \\
\hline & Intercept & 1.5051 & 0.4235 & 12.63 & 0.00 \\
\hline 1981 RECS & D1981 & -0.5834 & 0.2259 & 6.67 & 0.01 \\
\hline 1982 RECS & D1982 & -0.3602 & 0.2288 & 2.48 & 0.12 \\
\hline 1984 RECS & D1984 & -0.4220 & 0.2324 & 3.30 & 0.07 \\
\hline Cooling degree days & CDD65 & $-6.1 E-4$ & $1.4 E-4$ & 19.09 & 0.00 \\
\hline Heating degree days & HDD65 & $-1.9 E-4$ & 4.4E-5 & 17.62 & 0.00 \\
\hline Joint degree days & CDD65*Hdd65 & $4.2 E-7$ & $6.7 E-8$ & 39.05 & 0.00 \\
\hline Real income & INCONE & $2.0 E-5$ & $7.2 E-6$ & 11.47 & 0.00 \\
\hline Square feel heated & HEATED & $1.5 E-4$ & $1.2 E-4$ & 1.56 & 0.21 \\
\hline Attached single-family home & SINGFAM2 & $-2.3 E-5$ & 0.4859 & 0.00 & 1.00 \\
\hline 2-4 Multifamily units & MULTFAM1 & 0.4294 & 0.2933 & 2.14 & 0.14 \\
\hline$>4$ Multifamily units & MULTFAM2 & 0.2527 & 0.2474 & 1.04 & 0.31 \\
\hline Mobile homes & MOBULHOM & 0.6669 & 0.3006 & 4.92 & 0.03 \\
\hline Year nome built, $<1940$ & KHOUS1 & -0.6011 & 0.2831 & 4.51 & 0.03 \\
\hline Year home built, $1940-49$ & MHOUSE & -1.1651 & 0.3441 & 11.47 & 0.00 \\
\hline Year home built, 1960-74 & MHOUS4 & -1.1787 & 0.2581 & 20.36 & 0.00 \\
\hline Year home built, 1975-79 & KHOUS5 & -2.0925 & 0.2888 & 52.49 & 0.00 \\
\hline Year home built, $\geq 1980$ & KHOLS6 & -1.8364 & 0.3447 & 28.38 & 0.00 \\
\hline Central city location & INCITY & 0.7225 & 0.1689 & 18.30 & 0.00 \\
\hline Poor householder & POOA & -0.0198 & 0.2261 & 0.01 & 0.93 \\
\hline Hispanic origin & LATIN & 1.8344 & 0.3940 & 21.67 & 0.00 \\
\hline Black householder & RACE & 0.7060 & 0.4178 & 2.86 & 0.09 \\
\hline $\begin{array}{l}\text { Dependent variable: } \\
\text { Natural gas used } \\
\text { (Yes: NG=1, No: NG=0) }\end{array}$ & $N G$ & & & & \\
\hline Number of observations & 1,035 & & & & \\
\hline Log likelihood & $1,079.75$ & & & & \\
\hline
\end{tabular}


TABLE 16 Ordinary-Least-Squares-Parameter Estimates for Natural Gas Price Model: West Census Region

\begin{tabular}{|c|c|c|c|c|c|}
\hline Variable & $\begin{array}{l}\text { Variable } \\
\text { Code }\end{array}$ & $\begin{array}{l}\text { Parameter } \\
\text { Estimate }\end{array}$ & $\begin{array}{l}\text { Standard } \\
\text { Error }\end{array}$ & $\begin{array}{c}\mathrm{T} \text { for } \mathrm{HO}: \\
\text { Farameter }=0\end{array}$ & $\begin{array}{l}\text { Probabiiity } \\
\text { >Absolute } T\end{array}$ \\
\hline & Intercept & 1.6851 & 1.0191 & 1.65 & 0.09 \\
\hline 1982 RECS & D1982 & 0.6274 & 0.1709 & 3.67 & 0.00 \\
\hline 1984 RECS & D1984 & 0.8889 & 0.1609 & 5.53 & 0.00 \\
\hline Cooling degree days & CDD65 & $3.6 \mathrm{E}-4$ & $9.9 E-5$ & 3.65 & 0.00 \\
\hline Heating degree days & HDD65 & $-9.5 E-5$ & 3.7E-5 & -2.61 & 0.01 \\
\hline Consumer price index-urban & CPIU & 1.9438 & 0.6469 & 3.01 & 0.00 \\
\hline Square feet heated & HEATED & $1.6 \mathrm{E}-4$ & $9.8 E-5$ & 1.64 & 0.10 \\
\hline Attached single-family home & SINGFAM2 & -0.3081 & 0.3423 & -0.90 & 0.37 \\
\hline 2-4 Multifamily units & MULTFAM1 & 0.0487 & 0.2289 & 0.21 & 0.83 \\
\hline$>4$ Multifamily units & MULTFAM2 & 0.3039 & 0.2116 & 1.44 & 0.15 \\
\hline Mobile homes & MOBILHOM & 0.0616 & 0.3216 & 0.19 & 0.85 \\
\hline Year home built, $<1940$ & KHOUS1 & 0.2111 & 0.2137 & 0.99 & 0.32 \\
\hline Year home built, $1940-49$ & KHOUS2 & 0.9027 & 0.2903 & 3.11 & 0.00 \\
\hline Year home built, $1960-74$ & KHOUS4 & -0.0674 & 0.2149 & -0.31 & 0.75 \\
\hline Year home built, 1975-79 & KHOUS5 & -0.3088 & 0.3288 & -0.94 & 0.35 \\
\hline Year home built, $\geq 1980$ & KHOUS6 & -0.2684 & 0.3449 & -0.79 & 0.44 \\
\hline Central city location & INCITY & -0.0374 & 0.1612 & -0.23 & 0.82 \\
\hline Poor householder & POOR & -0.1022 & 0.1760 & -0.58 & 0.56 \\
\hline Hispanic origin & LATIN & -0.0715 & 0.2493 & -0.29 & 0.77 \\
\hline Black householder & RACE & -0.2168 & 0.2726 & -0.79 & 0.43 \\
\hline Mills ratio & m & -0.2572 & 0.2399 & -1.07 & 0.28 \\
\hline \multicolumn{6}{|l|}{ Dependent variable: } \\
\hline Natural gas price & PRICENG & & & & \\
\hline Number of observations & 3,650 & & & & \\
\hline Adjusted $\mathrm{A}^{2}$ & 0.0216 & & & & \\
\hline
\end{tabular}


TABLE 17 Maximum-Likelihood-Parameter Estimates for LPG Fuel-Share Model: Northeast Census Region

\begin{tabular}{|c|c|c|c|c|c|}
\hline Variable & $\begin{array}{l}\text { Variable } \\
\text { Code }\end{array}$ & $\begin{array}{c}\text { Parameter } \\
\text { Estimate }\end{array}$ & $\begin{array}{c}\text { Standard } \\
\text { Error: }\end{array}$ & Chi Square & Probability \\
\hline & Intercept & 0.4530 & 1.5276 & 0.09 & 0.77 \\
\hline 1981 RECS & D1981 & -0.2101 & 0.4127 & 0.26 & 0.61 \\
\hline 1982 RECS & D1982 & -0.5767 & 0.4709 & 1.50 & 0.22 \\
\hline 1984 RECS & D1984 & -0.0960 & 0.4124 & 0.05 & 0.82 \\
\hline Cooling degree days & CDD65 & -0.0018 & 0.0025 & 0.51 & 0.47 \\
\hline Heating degree days & HDD65 & $-8.4 E-5$ & 1.7E-4 & 0.24 & 0.62 \\
\hline Joint degree days & CDD65*Hdd65 & $-2.1 E-7$ & 4.3E-7 & 0.24 & 0.62 \\
\hline Real income & NCONE & $-3.8 E-5$ & $1.5 E-5$ & 6.57 & 0.01 \\
\hline Square feet heated & HEATED & $-2.4 E-5$ & $1.9 E-4$ & 0.02 & 0.90 \\
\hline 2-4 Multifamily units & MULTFAM1 & -0.7078 & 0.4645 & 2.32 & 0.13 \\
\hline$>4$ Multifamily units & MULTFAM2 & -1.0814 & 0.6650 & 2.64 & 0.10 \\
\hline Mobile homes & MOK3ILHOM & 2.4263 & 0.5404 & 20.16 & 0.00 \\
\hline Year home built, $<1940$ & KHOUS1 & 0.8880 & 0.4883 & 3.31 & 0.07 \\
\hline Year home built, $1940-49$ & KHOUS2 & -0.2147 & 0.7499 & 0.08 & 0.77 \\
\hline Year home built, 1960-74 & KHOUS4 & -0.2532 & 0.5593 & 0.21 & 0.65 \\
\hline Year home built, 1975-79 & KHOUS5 & -1.1078 & 0.8126 & 1.86 & 0.17 \\
\hline Year home built, $\geq 1980$ & KHCUS6 & -0.2255 & 0.8239 & 0.07 & 0.78 \\
\hline Central city location & INCITY & -2.5412 & 0.7444 & 11.65 & 0.00 \\
\hline Poor householder & POOR & -0.9681 & 0.4324 & 5.01 & 0.02 \\
\hline Hispanic origin & LATIN & 0.4490 & 0.6708 & 0.45 & 0.50 \\
\hline Black householder & RACE & -1.2168 & 1.0458 & 1.35 & 0.25 \\
\hline $\begin{array}{l}\text { Dependent variable: } \\
\text { LPG used } \\
\text { (Yes: LP=1, No: LP=0) }\end{array}$ & LP & & & & \\
\hline Number of observations & 1,234 & & & & \\
\hline Log likelihood & 420.07 & & & & \\
\hline
\end{tabular}


Table 18 shows the OLS-parameter Northwesi census region estimates for LPG price model. The CPIU and RACE (black householder) variables are significant at the 5\% level.

\subsubsection{Midwest Census Region}

Table 19 shows the LPG Midwest census region maximum-likelihood-parameter estimates for the fuel-share model. Variables significant at the 5\% level are mobile homes, central city location, homes built after 1979, homes built before 1940, homes built from 1960 through 1974, poor households, heated areas, and the 1981 RECS dummy variable.

Table 20 shows the OLS-parameter Midwest census region estimates for the LPG fuelprice model. The following variables are significant at the 5\% level: cooling degree days, the 1984 RECS dummy variable, and heating degree days.

\subsubsection{South Census Region}

Table 21 shows the LPG South census region maximum-likelihood-parameter estimates for the fuel-share model. The mobile homes, central city location, homes built from 1960 through 1979 , and black householders are significant at the 5\% level.

Table 22 shows the OLS-parameter South census region estimates for the LPG price model. All variables except the 1984 RECS dummy variable, the CPIU, area heated, attached single-family homes, and two-to-four multifamily units are significant at tie $5 \%$ level.

\subsubsection{West Census Region}

Table 23 shows LPG West census region maximum-likelihood-parameter estimates for the fuel-share model. The following variables are significant at the 5\% level: central city location, Hispanic ethnicity, and large multifamily units.

Table 24 shows the West census region OLS-parameter estimates for the LPG price model. Variables significant at the 5\% level include the CPIU, the 1984 RECS dummy variable, cooling degree days, and mobile homes. 
TABLE 18 Ordinary-Least-Squares-Parameter Estimates for LPG Price Model: Northeast Census Region

\begin{tabular}{|c|c|c|c|c|c|}
\hline Variable & $\begin{array}{l}\text { Variable } \\
\text { Code }\end{array}$ & $\begin{array}{c}\text { Parameter } \\
\text { Estimate }\end{array}$ & $\begin{array}{l}\text { Standard } \\
\text { Error }\end{array}$ & $\begin{array}{c}\mathrm{T} \text { for } \mathrm{HO}: \\
\text { Parameter }=0\end{array}$ & $\begin{array}{l}\text { Probability } \\
\text { >Absolute T }\end{array}$ \\
\hline & Intercept & -4.2227 & 3.6915 & -1.14 & 0.25 \\
\hline 1982 RECS & D1982 & .0 .2379 & 0.6883 & -0.35 & 0.73 \\
\hline 1984 RECS & D1984 & 0.7782 & 0.5004 & 1.56 & 0.12 \\
\hline Cooling degree days & CDD65 & $-5.4 E-4$ & 0.0021 & -0.26 & 0.80 \\
\hline Heating degree days & HDD65 & $3.0 \mathrm{E}-4$ & $2.4 \mathrm{E}-4$ & 1.25 & 0.21 \\
\hline Consumer price index-urban & CPIU & 9.8525 & 1.9786 & 4.98 & 0.00 \\
\hline Square feet heated & HEATED & $1.9 \mathrm{E}-4$ & 2.4E-4 & 0.77 & 0.44 \\
\hline Attached single-family home & SINGFAM2 & -0.6704 & 1.5661 & -0.43 & 0.67 \\
\hline 2-4 Multifamily units & MULTFAM1 & -1.2229 & 0.8339 & -1.47 & 0.14 \\
\hline$>4$ Multifamily units & MULTFAM2 & -2.2175 & 1.4938 & -1.48 & $0 .: 4$ \\
\hline Mobile homes & MOBILHOM & 2.2898 & 1.2703 & 1.80 & 0.07 \\
\hline Year home built, <1940 & KHOUS1 & 1.1666 & 0.8111 & 1.44 & 0.15 \\
\hline Year home built, 1940-49 & KHOUS2 & 0.7907 & 1.1253 & 0.70 & 0.48 \\
\hline Year home built, 1960-74 & KHOUS4 & 0.3943 & 0.8151 & 0.48 & 0.63 \\
\hline Year home built, 1975-79 & KHOUS5 & -1.0517 & 1.2424 & -0.85 & 0.40 \\
\hline Year nome built, $\geq 1980$ & KHOUS6 & 0.0537 & 1.2019 & 0.04 & 0.96 \\
\hline Central city location & INCITY & -2.0542 & 1.6192 & -1.27 & 0.21 \\
\hline Poor householder & POOR & -1.0924 & 0.6086 & -1.80 & 0.07 \\
\hline Hispanic origin & LATIN & 2.1533 & 1.6480 & 1.31 & 0.19 \\
\hline Black householder & RACE & -5.9133 & 2.9831 & -1.98 & 0.05 \\
\hline Mills ratio & m & -0.8257 & 0.4808 & -1.72 & 0.09 \\
\hline Dependent variable: & & & & & \\
\hline LPG price & PRICELP & & & & \\
\hline Number of observations & 353 & & & & \\
\hline Adjusted $\mathrm{R}^{2}$ & 0.0992 & & & & \\
\hline
\end{tabular}


TABLE 19 Maximum-Likelihood-Parameter Estimates for LPG Fuel-Share Model: Midwest Census Region

\begin{tabular}{|c|c|c|c|c|c|}
\hline Variable & $\begin{array}{c}\text { Variable } \\
\text { Code }\end{array}$ & $\begin{array}{l}\text { Parameter } \\
\text { Estimate }\end{array}$ & $\begin{array}{l}\text { Standard } \\
\text { Error }\end{array}$ & Chi Square & Probability \\
\hline & Intercept & -4.4725 & 1.1016 & 16.49 & 0.00 \\
\hline 1981 RECS & D1981 & 0.5375 & 0.2633 & 4.17 & 0.04 \\
\hline 1982 RECS & D1982 & -0.1261 & 0.3290 & 0.15 & 0.70 \\
\hline 1984 RECS & D1984 & -0.4141 & 0.2980 & 1.93 & 0.16 \\
\hline Cooling degree days & CDD65 & 0.0013 & $9.5 E-4$ & 1.92 & 0.17 \\
\hline Heating degree days & HDD65 & $7.0 E-5$ & $1.2 E-4$ & 0.33 & 0.57 \\
\hline Joint degree days & CDD65*Hdd65 & $-2.0 \mathrm{E}-7$ & $1.7 E-7$ & 1.309 & 0.25 \\
\hline Real income & INCONE & $9.9 E-7$ & $8.2 E-6$ & 0.01 & 0.90 \\
\hline Square feet heated & HEATED & $2.6 E-4$ & $1.1 E-4$ & 5.71 & 0.02 \\
\hline Attached single-family home & SINGFAM2 & 0.0786 & 0.5569 & 0.02 & 0.89 \\
\hline Mobile homes & MOBILHOM & 2.1184 & 0.3304 & 41.11 & 0.00 \\
\hline Year home built, $<1940$ & KHOUS1 & 1.7136 & 0.4916 & 12.15 & 0.00 \\
\hline Year home built, $1940-49$ & KHOUS2 & 1.1671 & 0.6062 & 3.71 & 0.05 \\
\hline Year home built, 1960-74 & KHOUS4 & 1.3332 & 0.4985 & 7.15 & 0.01 \\
\hline Year home built, 1975-79 & KHOUS5 & 0.9069 & 0.5477 & 2.74 & 0.10 \\
\hline Year home built, $\geq 1980$ & KHOUS6 & 2.1981 & 0.6291 & 12.21 & 0.00 \\
\hline Central city location & INCITY & -4.4399 & 1.0097 & 19.34 & 0.00 \\
\hline Poor householder & POOR & 0.6465 & 0.2608 & 6.14 & 0.01 \\
\hline Hispanic origir & LATIN & -1.3338 & 1.1020 & 1.46 & 0.23 \\
\hline $\begin{array}{l}\text { Dependent variabie: } \\
\text { LPG used } \\
\text { (Yes: } L P=1 \text {, No: } L P=0 \text { ) }\end{array}$ & IP & & & & \\
\hline Number of observations & 1,344 & & & & \\
\hline Log likelihood & 740.04 & & & & \\
\hline
\end{tabular}


TABLE 20 Ordinary-Least-Squares-Parameter Estimates for LPG Price Model: Midwest Census Region

\begin{tabular}{|c|c|c|c|c|c|}
\hline Variable & $\begin{array}{l}\text { Variable } \\
\text { Code }\end{array}$ & $\begin{array}{c}\text { Parameter } \\
\text { Estimate }\end{array}$ & $\begin{array}{l}\text { Standard } \\
\text { Error }\end{array}$ & $\begin{array}{c}\mathrm{T} \text { for } \mathrm{HO}: \\
\text { Parameter }=0\end{array}$ & $\begin{array}{l}\text { Probability } \\
\text { >Absolute } T\end{array}$ \\
\hline & Intercept & 16.2642 & 3.4274 & 4.75 & 0.00 \\
\hline 1982 RECS & D1982 & 0.5404 & 0.5927 & 0.91 & 0.36 \\
\hline 1984 RECS & D1984 & 1.8108 & 0.7222 & 2.51 & 0.01 \\
\hline Cooling degree days & CDD65 & $-2.8 E-3$ & $6.6 \mathrm{E}-4$ & -4.31 & 0.00 \\
\hline Heating degree days & HDD65 & $-3.1 E-4$ & $1.3 E-4$ & -2.49 & 0.01 \\
\hline Sonsumer price index-urban & CPIU & 2.4365 & 2.4812 & 0.98 & 0.33 \\
\hline Square feet heated & HEATED & $-5.5 E-4$ & $3.0 \mathrm{E}-4$ & -1.81 & 0.07 \\
\hline Attached sing!o-family home & SINGFAM2 & -0.2769 & 0.9851 & -0.28 & 0.78 \\
\hline 2-4 Multifamily units & MULTFAM1 & 0.9744 & 1.5751 & 0.62 & 0.54 \\
\hline$>4$ Multifamily units & MULTFAM2 & 3.7563 & 2.3512 & 1.60 & 0.11 \\
\hline Mobile homes & MOBILHOM & -2.1276 & 2.0864 & -1.02 & 0.31 \\
\hline Year home built, <1940 & KHOUS1 & -2.2039 & 1.9202 & -1.15 & 0.25 \\
\hline Year home built, $1940-49$ & KHOUS2 & 0.0104 & 1.4741 & 0.01 & 0.99 \\
\hline Year home built, 1960-74 & KHOUS4 & .1 .8358 & 1.5596 & -1.18 & 0.24 \\
\hline Year home built, 1975-79 & KHOUS5 & -1.1414 & 1.2114 & -0.94 & 0.35 \\
\hline Year home built, $\geq 1980$ & KHOUS6 & -2.1012 & 2.4097 & -0.87 & 0.38 \\
\hline Central city location & INCITY & 6.5828 & 5.2216 & 1.26 & 0.21 \\
\hline Poor householder & POOR & -0.0439 & 0.7362 & -0.06 & 0.95 \\
\hline Hispanic origin & LATIN & 0.8246 & 1.6066 & 0.51 & 0.61 \\
\hline Mills ratio & m & 1.4568 & 1.1572 & 1.26 & 0.21 \\
\hline Dependent variable: & & & & & \\
\hline LPG price & PRICELP & & & & \\
\hline Number of observations & 610 & & & & \\
\hline Adjusted $\mathrm{R}^{2}$ & 0.0731 & & & & \\
\hline
\end{tabular}


TABLE 21 Maximum-Likelihood-Parameter Estimates for LPG Fuel-Share Model: South Census Region

\begin{tabular}{|c|c|c|c|c|c|}
\hline Variable & $\begin{array}{l}\text { Variable } \\
\text { Code }\end{array}$ & $\begin{array}{c}\text { Parameter } \\
\text { Estimate }\end{array}$ & $\begin{array}{l}\text { Standard } \\
\text { Error }\end{array}$ & Chi Square & Probability \\
\hline & Intercept & -0.9939 & 1.1525 & 0.74 & 0.39 \\
\hline 1981 RECS & D1981 & 0.2287 & 0.2861 & 0.64 & 0.42 \\
\hline 1982 RECS & D1982 & 0.0091 & 0.3358 & 0.00 & 0.98 \\
\hline 1984 RECS & D1984 & -0.1444 & 0.3151 & 0.21 & 0.65 \\
\hline Cooling degree days & CDD65 & $-2.1 E-4$ & 3.1E-4 & 0.46 & 0.50 \\
\hline Heating degree days & HDD65 & $-3.4 E-4$ & 2.0E-4 & 2.86 & 0.09 \\
\hline Joint degree days & CDD65*Hdd65 & $9.2 E-8$ & 8.6E-8 & 1.15 & 0.28 \\
\hline Real income & INCOME & $-2.2 E-6$ & 1.1E-5 & 0.04 & 0.84 \\
\hline Square feet heated & HEATED & 1.3E-4 & $1.6 \mathrm{E}-4$ & 0.60 & 0.44 \\
\hline Attached single-family home & SINGFAM2 & -0.4997 & 1.0478 & 0.23 & 0.63 \\
\hline Mobile homes & MOBILHOM & 2.3855 & 0.3113 & 58.71 & 0.00 \\
\hline Year home built, $<1940$ & KHOUS1 & 0.5303 & 0.3303 & 2.58 & 0.11 \\
\hline Year home built, $1940-49$ & KHOUS2 & -0.5534 & 0.4266 & 1.68 & 0.19 \\
\hline Year home built, 1960-74 & KHOUS4 & -1.0470 & 0.3320 & 9.95 & 0.00 \\
\hline Year home built, 1975-79 & KHOUS5 & -0.9706 & 0.4252 & 5.21 & 0.02 \\
\hline Year home built, $\geq 1980$ & KHOUS6 & -0.8341 & 0.4690 & 3.16 & 0.08 \\
\hline Central city location & INCITY & -3.0211 & 0.5308 & 32.39 & 0.00 \\
\hline Poor householder & POOR & 0.3516 & 0.2677 & 1.72 & 0.19 \\
\hline Hispanic origin & LATIN & 0.6275 & 0.6321 & 0.99 & 0.32 \\
\hline Black householder & RACE & 0.4960 & 0.2508 & 3.91 & 0.05 \\
\hline $\begin{array}{l}\text { Dependent variable: } \\
\text { LPG used }\end{array}$ & $\mathbf{P}$ & & & & \\
\hline (Yes: LP=1, No: LP=0) & & & & & \\
\hline Number of observations & 1,173 & & & & \\
\hline Log likelihood & 641.05 & & & & \\
\hline
\end{tabular}


TABLE 22 Ordinary-Least-Squares-Parameter Estimates for LPG Price Model: South Census Region

\begin{tabular}{|c|c|c|c|c|c|}
\hline Variable & $\begin{array}{l}\text { Variable } \\
\text { Code }\end{array}$ & $\begin{array}{l}\text { Parameter } \\
\text { Estimate }\end{array}$ & $\begin{array}{l}\text { Standard } \\
\text { Error }\end{array}$ & $\begin{array}{c}\mathbf{T} \text { for } \mathrm{HO}: \\
\text { Parameter }=0\end{array}$ & $\begin{array}{l}\text { Probability } \\
\text { >Absolute } T\end{array}$ \\
\hline & Intercept & -4.1611 & 1.9151 & -2.17 & 0.03 \\
\hline 1982 RECS & D1982 & -0.7551 & 0.3111 & -2.43 & 0.02 \\
\hline 1984 RECS & D1984 & 0.1441 & 0.3020 & 0.48 & 0.63 \\
\hline Cooling degree days & CDD65 & 0.0015 & $3.3 E-4$ & 4.72 & 0.00 \\
\hline Heating degree days & HDD65 & -0.0010 & $2.1 E-4$ & -4.81 & $0 . \mathrm{CO}$ \\
\hline Consumer price index-urban & CPIU & 1.0352 & 1.0881 & 0.95 & 0.34 \\
\hline Square feet heated & HEATED & 4.1E-4 & 2.3E-4 & 1.81 & 0.07 \\
\hline Attached single-family home & SINGFAM2 & -2.0032 & 2.1867 & -0.92 & 0.36 \\
\hline 2-4 Multifamily units & MULTFAM1 & 4.3106 & 3.0618 & 1.41 & 0.16 \\
\hline$>4$ Multifamily units & MULTFAM2 & 9.3984 & 3.0898 & 3.04 & 0.00 \\
\hline Mobile homes & MOBILHOM & 12.1873 & 1.1165 & 10.92 & 0.00 \\
\hline Year home built, $<1940$ & KHOUS1 & 2.5931 & 0.4966 & 5.22 & 0.00 \\
\hline Year home built, 1940-49 & KHOUS2 & -2.7358 & 0.5077 & -5.39 & 0.00 \\
\hline Year home built, 1960-74 & KHOUS4 & -5.3867 & 0.5804 & -9.28 & 0.00 \\
\hline Year home built, 1975-79 & KHOUS5 & -6.1060 & 0.6051 & -10.09 & 0.00 \\
\hline Year home built, $\geq 1980$ & KHOUS6 & -5.3232 & 0.6126 & -8.69 & 0.00 \\
\hline Central city location & INCITY & -13.0327 & 1.6800 & -7.76 & 0.00 \\
\hline Poor householder & POOR & 1.7690 & 0.2995 & 5.91 & 0.00 \\
\hline Hispanic origin & LATIN & 1.4758 & 0.6941 & 2.13 & 0.03 \\
\hline Black householder & RACE & 2.6173 & 0.3730 & 7.02 & 0.00 \\
\hline Mills ratio & $\mathbf{m}$ & -5.9680 & 0.6213 & -9.61 & 0.00 \\
\hline Dependent variable: & & & & & \\
\hline LPG price & PRICELP & & & & \\
\hline Number of observations & 820 & & & & \\
\hline Adjusted $R^{2}$ & 0.2713 & & & & \\
\hline
\end{tabular}


TABLE 23 Maximum-Likelihood-Parameter Estimates for LPG Fuel-Share Model: West Census Region

\begin{tabular}{|c|c|c|c|c|c|}
\hline Variable & $\begin{array}{l}\text { Variable } \\
\text { Code }\end{array}$ & $\begin{array}{c}\text { Parameter } \\
\text { Estimate }\end{array}$ & $\begin{array}{l}\text { Standard } \\
\text { Error }\end{array}$ & Chi Square & Probability \\
\hline & Interçept & -2.4908 & 0.9554 & 6.80 & 0.01 \\
\hline 1981 RECS & D1981 & -0.3470 & 0.4717 & 0.54 & 0.46 \\
\hline 1982 RECS & Di982 & -0.0751 & 0.4713 & 0.03 & 0.87 \\
\hline 1984 RECS & D1984 & -0.3927 & 0.4996 & 0.62 & 0.43 \\
\hline Cooling degree days & CDD65 & $3.1 E-4$ & 3.0E-4 & 1.10 & 0.29 \\
\hline Heating degree days & HDD65 & $1.6 E-4$ & 9.9E-5 & 2.69 & 0.10 \\
\hline Joint degree days & CDD65*Hdd65 & 2.1E-8 & 1.1E-7 & 0.04 & 0.85 \\
\hline Real income & INCOME & $-3.6 E-5$ & 2.2E-5 & 2.74 & 0.10 \\
\hline Square feet heated & HEATED & $-4.8 E-4$ & 3.OE-4 & 2.56 & 0.11 \\
\hline Attached single family home & SINGFAM2 & -0.2519 & 1.0927 & 0.05 & 0.82 \\
\hline 2-4 Multifamily units & MULTFAM1 & -1.2179 & 0.7909 & 2.37 & 0.12 \\
\hline$>4$ Multifamily units & MULTFAM2 & -2.2168 & 1.0687 & 4.30 & 0.04 \\
\hline Mobile homes & MOBILHOM & 0.1228 & 0.5018 & 0.06 & 0.81 \\
\hline Year home built, $<1940$ & KHOUS1 & 0.3584 & 0.5667 & 0.40 & 0.53 \\
\hline Year home built, 1940-49 & KHOUS2 & 1.0811 & 0.6190 & 3.05 & 0.08 \\
\hline Year home built, 1960-74 & KHOUS4 & 0.7185 & 0.5278 & 1.85 & 0.17 \\
\hline Year home built, 1975-79 & KHOUS5 & -0.3821 & 0.7669 & 0.25 & 0.62 \\
\hline Year home buill, $\geq 1980$ & KHOUS6 & -0.0623 & 0.8916 & 0.00 & 0.94 \\
\hline Central city location & INCITY & -3.2735 & 1.0235 & 10.23 & 0.00 \\
\hline Poor householder & POOR & 0.5104 & 0.4217 & 1.47 & 0.23 \\
\hline Hispanic origin & LATIN & -2.2680 & 1.0447 & 4.71 & 0.03 \\
\hline $\begin{array}{l}\text { Dependent variable: } \\
\text { LPG used } \\
\text { (Yes: } L P=1 \text {, No: } L P=0 \text { ) }\end{array}$ & LP & & & & \\
\hline $\begin{array}{l}\text { Number of observations } \\
\text { Log likelihood }\end{array}$ & $\begin{array}{l}1035 \\
300.54\end{array}$ & & & & \\
\hline
\end{tabular}


TABLE 24 Ordinary-Least-Squares-Parameter Estimates for LPG Price Model: West Census Region

\begin{tabular}{|c|c|c|c|c|c|}
\hline Variable & $\begin{array}{l}\text { Variable } \\
\text { Code }\end{array}$ & $\begin{array}{l}\text { Parameter } \\
\text { Estimate }\end{array}$ & $\begin{array}{l}\text { Standard } \\
\text { Error }\end{array}$ & $\begin{array}{c}T \text { for } \mathrm{HO}: \\
\text { Parameter }=0\end{array}$ & $\begin{array}{l}\text { Probability } \\
\text { >Absolute } T\end{array}$ \\
\hline & Intercept & 0.5265 & 5.0062 & 0.10 & 0.92 \\
\hline 1982 RECS & D1982 & 0.8051 & 0.6725 & 1.20 & 0.23 \\
\hline 1984 RECS & D1984 & 1.6590 & 0.6223 & 2.67 & 0.01 \\
\hline Cooling degree days & CDD65 & $1.0 \mathrm{E}-3$ & 4.5E-4 & 2.29 & 0.02 \\
\hline Heating degree days & HDD65 & $-3.7 E-4$ & $2.0 E-4$ & -1.86 & 0.06 \\
\hline Consumer price index-urban & CPIU & 7.7846 & 2.5375 & 3.07 & 0.00 \\
\hline Square feet heated & HEATED & $-5.9 E-4$ & $4.4 E-4$ & -1.34 & 0.18 \\
\hline Attached single-family home & SINGFAMR & -1.8151 & 3.6513 & -0.50 & 0.62 \\
\hline 2-4 Multifamily units & MULTFAM1 & -1.2921 & 2.1888 & -0.59 & 0.56 \\
\hline$>4$ Multifamily units & MULTFAM2 & -0.6040 & 3.9567 & .0 .15 & 0.88 \\
\hline Mobile homes & MOBILHOM & -1.6363 & 0.7489 & -2.19 & 0.03 \\
\hline Year home built, <1940 & KHOUS1 & -0.9746 & 0.8513 & -1.14 & 0.25 \\
\hline Year home built, $1940-49$ & KHOUS2 & -1.3211 & 1.1659 & -1.13 & 0.26 \\
\hline Year home built, $1960-74$ & KHOUS4 & -0.7423 & 0.8253 & -0.90 & 0.37 \\
\hline Year home built, $1975-79$ & KHOUS5 & -0.9300 & 1.0579 & -0.88 & 0.38 \\
\hline Year home built, $\geq 1980$ & KHOUS6 & -0.1043 & 1.2353 & -0.08 & 0.93 \\
\hline Central city location & INCITY & -2.4056 & 2.3112 & -1.04 & 0.30 \\
\hline Poor householder & POCR & 1.1792 & 0.7570 & 1.56 & 0.12 \\
\hline Hispanic origin & LATIN & -1.7577 & 1.6814 & -1.05 & 0.30 \\
\hline Milis ratio & m & -0.3873 & 0.5892 & -0.66 & 0.51 \\
\hline Dependent variable: & & & & & \\
\hline LPG price & PRICELP & & & & \\
\hline Number of observations & 232 & & & & \\
\hline Adjusted $\mathrm{R}^{2}$ & 0.3130 & & & & \\
\hline
\end{tabular}




\section{Overview of Fuel-Share Models}

Twelve fuel-share models (three fuel types and four census regions) were introduced in Section 3. Up to 21 explanatory variables were included in the models. Table 25 shows significant variables at the 5\% level for each of the 12 models. The following three variables are significant at the 5\% level in at least half of these models: the central city location (8 out of 12), mobile homes ( 7 out of 12), and homes built after 1979 (6 out of 12). Other highlights are discussed below.

The probability of using natural gas in all census regions is greater in central city locations than in locations outside the central city. The opposite is true for LPG, which has a greater use in locations outside the sentral city.

The probability of black households using natural gas in Northeast, Midwest, and South census regions is significantly (5\% level) higher than that of the nonblack households. The Hispanic households also have higher probability of using natural gas, compared to non-Hispanic households, but only in the South and West census regions.

The probability of using fuel oil/kerosine in mobile homes in the Northeast and South census regions is significantly ( $5 \%$ level) higher than that in nonmobile homes.

The probability of using fuel oil/kerosine in homes built after 1979 in the Northeast and South census regions is significantly (5\% level) iv wer than that in homes built before 1980 .

The probability of using LPG in mobile homes in the Northeast, Midwest, and South census regions is significantly ( $5 \%$ level) higher than that in nonmobile homes. 
TABLE 25 Key Variables Significant at 5\% Level in Fuel-Strare Models

\begin{tabular}{|c|c|c|c|c|c|c|c|c|c|c|c|c|}
\hline \multirow[b]{2}{*}{ Variable } & \multicolumn{4}{|c|}{ Fuel Oil/Kerosine } & \multicolumn{4}{|c|}{ Natural Gas } & \multicolumn{4}{|c|}{ LPG } \\
\hline & NE & MMN & so & WE & NE & $\mathbf{M} \mathbf{N}$ & so & WE & NE & $\mathbf{M W}$ & so & WE \\
\hline Intercept & & & & $x$ & & $x$ & $x$ & $x$ & & $x$ & & $x$ \\
\hline 1981 RECS (D1981) & & $x$ & $x$ & & $x$ & & & $x$ & & $x$ & & \\
\hline $\begin{array}{l}1982 \text { RECS (D1982) } \\
1984 \text { RECS (D1984) }\end{array}$ & & $x$ & $x$ & & $x$ & & $\mathbf{x}$ & & & & & \\
\hline $\begin{array}{l}\text { Cooling degree days } \\
\text { (CDD65) }\end{array}$ & & & & $x$ & & $x$ & & $x$ & & & & \\
\hline $\begin{array}{l}\text { Heating degree days } \\
\text { (HDD65) }\end{array}$ & & & $x$ & & $x$ & $x$ & & $x$ & & & & \\
\hline $\begin{array}{l}\text { Joint degree days } \\
\text { (CDD65*Hdd65) }\end{array}$ & & & $x$ & & $x$ & $x$ & $x$ & $x$ & & & & \\
\hline Real income (INCOME) & $x$ & & & $x$ & $x$ & & & $x$ & $x$ & & & \\
\hline $\begin{array}{l}\text { Square feet heated } \\
\text { (HEATED) }\end{array}$ & & & & $x$ & $x$ & & & & & $x$ & & \\
\hline $\begin{array}{l}\text { Attached single-family } \\
\text { home (SINGFAM2) }\end{array}$ & $x$ & & & & & & & & & & & \\
\hline $\begin{array}{l}\text { 2-4 Multifamily units } \\
\text { (MULTFAM1) }\end{array}$ & $\mathbf{x}$ & & & & $x$ & $x$ & $x$ & & & & & \\
\hline $\begin{array}{l}>4 \text { Multifamily units } \\
\text { (MULTFAM2) }\end{array}$ & & $x$ & & & $x$ & $x$ & & & & & & $x$ \\
\hline Mobile home (MOBILHOM) & $x$ & & $\mathbf{x}$ & & & & $x$ & $x$ & $x$ & $\mathbf{x}$ & $\mathbf{x}$ & \\
\hline $\begin{array}{l}\text { Year home built, }<1940 \\
\text { (KHOUS1) }\end{array}$ & & & & & $x$ & $x$ & & $x$ & & $x$ & & \\
\hline $\begin{array}{l}\text { Year home built, 1940-49 } \\
\text { (KHOUS2) }\end{array}$ & & & & & $x$ & & & $x$ & & $x$ & & \\
\hline $\begin{array}{l}\text { Year home built, 1960-74 } \\
\text { (KHOUS4) }\end{array}$ & $x$ & & & & & $x$ & & $x$ & & $x$ & $x$ & \\
\hline $\begin{array}{l}\text { Year home built, } 1975-79 \\
\text { (KHOUS5) }\end{array}$ & & & & & $x$ & $x$ & $x$ & $x$ & & & $x$ & \\
\hline $\begin{array}{l}\text { Year home built, } \geq 1980 \\
\text { (KHOUS6) }\end{array}$ & $x$ & & $x$ & & & $x$ & $x$ & $x$ & & $x$ & & \\
\hline Central city(INCITY) & & & & & $x$ & $x$ & $x$ & $x$ & $x$ & $x$ & $x$ & $x$ \\
\hline Poor (POOR) & & & & & & & & & $x$ & $x$ & & \\
\hline Hispanics (LATIN) & $x$ & & & & & & $x$ & $x$ & & & & $x$ \\
\hline Blacks (RACE) & & & & & $x$ & $x$ & $x$ & & & & $x$ & \\
\hline
\end{tabular}




\section{Overview of Fuel-Price Models}

Twelve household fuel price models were introduced in Section 3. Twenty explanatory variables were included in each of the models. Table 26 shows significant variables at the $5 \%$ level for these models. The following seven variables are significant at the $5 \%$ level in at least half of the models: 1984 RECS dummy variable in 11 models, CPIU in 10, 1982 RECS dummy variable in 8, cooling degree days in 8, large multifamily units in 7, homes built from 1960 through 1974 in 6 , and central city location in 6 . Other highlights are discussed below.

The fuel prices paid by households in 1984 were significantly higher (at 5\% level) than pre1984 prices in 11 of the 12 models, and prices paid in 1982 were higher than pre-1982 prices in 8 of the 12 models.

Higher inflation as measured by CPI resulted in (1) relatively lower fuel oil/kerosine prices in all census regions and (2) higher natural gas prices in all regions, except the Northeast.

Households living in cold climates, as measured by HDD, are expected to consume more fuel for space and water heating. All else being equal, this should result in lower average fuel prices. Wherever the coefficient associated with HDD is significant at the $5 \%$ level, it has a negative sign. This occurs for natural gas prices paid by households living in the Northeast, Midwest, and West census regions and for LPG prices in the Midwest and South census regions.

Wherever the coefficient associated with the Hispanic dummy variable is significant at the 5\% level, it has a positive sign, implying that the fuel prices paid by Hispanics are higher than those paid by non-Hispanic households. This significance occurs for natural gas prices paid by Hispanic households living in the Northeast and Midwest census regions, for fuel oil/kerosine prices in the Midwest and West census regions, and for LPG prices in the South census region.

Coefficients associated with the black race dummy variable in fuel oil/kerosine, natural gas, and LPG price models for the South census region are significant at the 5\% level and have a positive sign. This implies that the fuel prices paid by blacks are higher than those paid by nonblacks in the South region. According to the information in a DOE/EIA report on the 1987 RECS, ${ }^{6}$ of the total national population, abcut $57 \%$ of the black households were in the South region, compared to only $32 \%$ of the white households. The cause of higher prices paid by black ho'seholds in this region is a subject for future research.

The LPG prices paid by households living either in attached single-family homes or in twoto-four multifamily homes in any census region were not significantly different at the 5\% level than those paid by households in the remaining home categories. On the other hand, other fuel (natural gas or fuel oil/kerosine) prices paid by households living either in attached single-family homes or in two-to-four multifamily homes were higher than the prices paid by other households. 
TABLE 26 Key Variables Significant at 5\% Level in Fuel-Price Models

\begin{tabular}{|c|c|c|c|c|c|c|c|c|c|c|c|c|}
\hline \multirow[b]{2}{*}{ Variable } & \multicolumn{4}{|c|}{ Fuel Oil/Kerosine } & \multicolumn{4}{|c|}{ Natural Gas } & \multicolumn{4}{|c|}{ LPG } \\
\hline & NE & $M W$ & so & WE & NE & $M W$ & so & WE & NE & $M W$ & so & WE \\
\hline Intercept & $x$ & $x$ & $x$ & $x$ & $x$ & & $x$ & & & $x$ & $x$ & \\
\hline 1982 RECS (D1982) & $x$ & $x$ & & $x$ & $x$ & $x$ & $x$ & $x$ & & & $x$ & \\
\hline 1984 RECS (D1984) & $x$ & $x$ & $x$ & $x$ & $x$ & $x$ & $x$ & $x$ & & $x$ & $x$ & $x$ \\
\hline $\begin{array}{l}\text { Cooling degree days } \\
\text { (CDD65) }\end{array}$ & & $x$ & & & $x$ & $x$ & $x$ & $x$ & & $x$ & $x$ & $x$ \\
\hline $\begin{array}{l}\text { Heating degree days } \\
\text { (HDD65) }\end{array}$ & & & & & $x$ & $x$ & & $x$ & & $x$ & $x$ & \\
\hline $\begin{array}{l}\text { CPIU } \\
\text { Square feet heated } \\
\text { (HEATED) }\end{array}$ & $x$ & $x$ & $x$ & $\mathbf{x}$ & $x$ & $x$ & $\begin{array}{l}x \\
x\end{array}$ & $x$ & $x$ & & & $x$ \\
\hline $\begin{array}{l}\text { Attached single-family } \\
\text { home (SINGFAM2) }\end{array}$ & $x$ & $x$ & & & & & $x$ & & & & & \\
\hline $\begin{array}{l}2-4 \text { Multifamily units } \\
\text { (MULTFAM1) }\end{array}$ & & $x$ & & & $\mathbf{x}$ & $x$ & $\mathbf{x}$ & & & & & \\
\hline $\begin{array}{l}>4 \text { Multifamily units } \\
\text { (MULTFAM2) }\end{array}$ & $x$ & $x$ & $x$ & & $x$ & $x$ & $x$ & & & & $x$ & \\
\hline Mobile home (MOBILHOM) & $x$ & & & $x$ & & & $x$ & & & & $x$ & $x$ \\
\hline $\begin{array}{l}\text { Year home built, <1940 } \\
\text { (KHOUS1) }\end{array}$ & & & $x$ & & $x$ & $x$ & $x$ & & & & $x$ & \\
\hline $\begin{array}{l}\text { Year home built, 1940-49 } \\
\text { (KHOUS2) }\end{array}$ & & $x$ & & & & & $x$ & $x$ & & & $x$ & \\
\hline $\begin{array}{l}\text { Year home built, 1960-74 } \\
\text { (KHOUS4) }\end{array}$ & $x$ & $x$ & $x$ & & $x$ & $x$ & & & & & $x$ & \\
\hline $\begin{array}{l}\text { Year home built, 1975-79 } \\
\text { (KHOUS5) }\end{array}$ & & & $x$ & $x$ & & $x$ & $x$ & & & & $x$ & \\
\hline $\begin{array}{l}\text { Year home built, } \geq 1980 \\
\text { (KHOUS6) }\end{array}$ & & & $x$ & $x$ & & $\mathbf{x}$ & $x$ & & & & $\mathbf{x}$ & \\
\hline Central city(INCITY) & $x$ & $x$ & & & $x$ & $x$ & $x$ & & & & $x$ & \\
\hline Poor (POOA) & & & & & & & $x$ & & & & $x$ & \\
\hline Hispanics (LATIN) & & $x$ & & $x$ & & $x$ & $x$ & & & & $x$ & \\
\hline Blacks (RACE) & & & $x$ & & & & $x$ & & $x$ & $y$ & $x$ & $y$ \\
\hline Mills Ratio (m) & & $x$ & & $x$ & & $x$ & $x$ & & & & $x$ & \\
\hline
\end{tabular}

Note: $x=$ significant variable; $y=$ variable not used in the model. 
The selectivity correction term (mills ratio) was found to be significant at the $5 \%$ level in 5 of the 12 cases (Table 26). The magnitude of the mills ratio $(\mathrm{m})$ declines with the increase in probability (p) of choosing a fuel (see Section 3, Equations 1 to 4). (For example, the value of mills ratio is calculated to be -3.97 for 0.05 probability, -3.25 for 0.1 probability, and -1.39 for 0.5 probability.) Therefore, the magnitude of the correction term increases with a decline in the probability of choosing a fuel. The magnitude and sign of the estimated coefficient $\left(d_{n+1}\right)$ of the mills ratio is important as well. The total adjustment is determined by the product of the mills ratio (m) and its coefficient $\left(d_{n+1}\right)$. In one of the five significant cases above, the coefficient $\left(d_{n+1}\right)$ is estimated to be positive $(+1.51)$ for fuel oil/kerosine price in the Midwest census region, implying a negative adjustment for correcting self-selection bias. For the other four significant cases, the coefficient $\left(d_{n+1}\right)$ is estimated to be negative. Specifically, the coefficient $\left(d_{n+1}\right)$ is estimated to be -0.29 for fuel oil/kerosine price in the West census region, -0.64 for natural gas in the Midwest, -1.96 for natural gas in the South, and -5.96 for LPG in the South. Among all of the 12 cases, the prices for LPG in the South census region are adjusted most (upward) for correction of selfselection bias. 


\section{Conclusions}

In this report, individual fuel prices are estimated within the residential sector, taking into account any required possible correction of self-selection bias. Estimates obtained by using only data in which observed fuel prices are present will be biased. For the important fuel types - fuel oil/kerosine, natural gas, and LPG - the estimation presents a self-selectivity bias problem because these fuels are not used by all households. Our literature search identified no past studies on the application of any selectivity model (which corrects for self-selection bias) for estimating household prices for fuel oil/kerosine, natural gas, and LPG.

The procedure for estimating models with correction for selection bias was adapted from the Conditional Expectation Correction Method described by Dubin and McFadden 1984. ${ }^{1}$ The selectivity models were developed for estimating prices of residential fuel oil/kerosine, natural gas, and LPG in the Northeast, Midwest, South, and West census regions. Statistically significant explanatory variables were identified and discussed in each of the models. This new application of the selectivity model should be of interest to energy policy makers, researchers, and academicians.

\subsection{Fuel-Share Models}

The following variables in the fuel-share models were found to be significant at the 5\% level in at least half of the 12 models: central city location ( 8 out of 12 models), mobile homes (7 out of 12), and homes built after 1979 (6 out of 12).

Some fuel-share models that relate to ethnicity/race are of special importance to the underlying research. The probability of black households using natural gas in the Northeast, Midwest, and South census regions is significantly ( $5 \%$ level) higher than that of the nonblack households. The Hispanic households also have a higher probability of using natural gas compared to non-Hispanic households, but only in the South and West census regions.

\subsection{Fuel-Price Models}

The following variables are significant at the 5\% level in at least half of the fuel-price models: 1984 RECS dummy variable in 11 models, CPIU in 10, the 1982 RECS dummy variable in 8, cooling degree days in 8, large multifamily units in 7, homes built from 1960 through 1974 in 6 , and central city location in 6 .

The selectivity correction term (mills ratio) was found to be significant at the $5 \%$ level in five of the 12 fuel-price cases. The prices for LPG in the South census region are adjusted most (upward) for correction of self-selection bias. 
Wherever the coefficient associated with the Hispanic dummy variable is significant at the 5\% level, it has a positive sign; this implies that the fuel prices paid by Hispanics are higher than those paid by non-Hispanic households. This significance occurs for ratural gas prices paid by Hispanic households living in the Northeast and Midwest census regions, fuel oil/kerosine prices in the Midwest and West census regions, and LPG prices in the South census region.

Coefficients associated with the black households (RACE) dummy variable in fuel oil/kerosine, natural gas, and LPG price models for the South census region are significant at the $5 \%$ level and have a positive sign. This implies that the fuel prices paid by blacks are higher than those paid by nonblacks in the South region. 


\section{References}

1. Dubin, J., and D. McFadden, 1984, "An Econometric Analysis of Residential Electric Appliance Holdings and Consumptions," Econometrica, 52:345-362.

2. U.S. Department of Energy, 1990, Residential Energy Consumption Survey 1981, public-use data tape, Energy Information Administration, Washington, D.C.

3. U.S. Department of Energy, 1990, Residential Energy Consumption Survey 1982, public-use data tape, Energy Information Administration, Washington, D.C.

4. U.S. Department of Energy, 1990, Residential Energy Consumption Survey 1984, public-use data tape, Energy Information Administration, Washington, D.C.

5. U.S. Department of Energy, 1990, Residential Energy Consumption Survey 1987, public-use data tape, Energy Information Administration, Washington, D.C.

6. U.S. Department of Energy, 1989, Housing Characteristics, 1987, report DOE/EIA-0314, Energy Information Administration, Washington, D.C.

7. U.S. Department of Energy, 1989, Household Energy Consumption and Expenditures, 1987; Part 1: National Data, report DOE/EIA-0321, Energy Information Administration, Washington, D.C.

8. Heckman, J.J., 1979, "Sample Selection Bias as a Specification Error," Econometrica, 47(1):153-162.

9. Maddala, G.S., 1983, Limited-Dependent and Qualitative Variables in Econometrics, Cambridge University Press, N.Y.

10. Engile, R.F., 1984, "Wald, Likelihood Ratio, and Lagrange Multiplier Tests in Econometrics," chap. 13, vol. 2, Handbook of Econometrics, Z. Griliches and M. Intriligator (editors), North-Holland, Inc., N.Y.

11. Dubin, J., and D. Rivers, 1989 and 1990, "Selection Bias in Linear Regression, Logit, and Probit Models," Sociological Methods and Research, vol. 18, nos. 2 and 3 (Nov. 1989 and Feb. 1990), pp. 360-390.

12. SAS Institute Inc., 1987, Software Release 6.04, Cary, N.C. 